Cahiers de recherches médiévales siècle)

\title{
Monachus non doctoris, sed plangentis habet officium
}

L'autorité de Jérôme dans le débat sur l'enseignement des moines aux XI et $\mathrm{XII}^{\mathrm{e}}$ siècles

\section{Thierry Kouamé}

\section{(2) OpenEdition \\ Journals}

Édition électronique

URL : https://journals.openedition.org/crm/11681

DOI : $10.4000 / \mathrm{crm} .11681$

ISSN : 1955-2424

Éditeur

Honoré Champion

\section{Édition imprimée}

Date de publication : 20 novembre 2009

Pagination : 9-38

ISSN : $1272-9752$

\section{Référence électronique}

Thierry Kouamé, "Monachus non doctoris, sed plangentis habet officium», Cahiers de recherches médiévales [En ligne], 18 | 2009, mis en ligne le 15 décembre 2012, consulté le 15 décembre 2022. URL : http://journals.openedition.org/crm/11681 ; DOI : https://doi.org/10.4000/crm.11681 


\title{
酶M
}

\section{Monachus non doctoris, sed plangentis habet officium L'autorité de Jérôme dans le débat sur l'enseignement des moines aux $\mathrm{XI}^{\mathrm{e}}$ et $\mathrm{XII}^{\mathrm{e}}$ siècles}

\begin{abstract}
While the controversy over monks' preaching and teaching was raging, St Jerome's maxim "the function of a monk is to weep not teach" was extremely influential. This aphorism, which spread through Tuscany from the end of the 11th century, had the advantage of being by a monk whose authority could not be questioned by the defenders of monastic order. From the circles of secular reformers it spread via the canonical collections to Italy and then to France; Ivo of Chartres popularized it. Jerome's maxim was subsequently adopted by theologians, and used in the ecclesiological debates of the early-12th century. But by mid-century, due to the influence of scholastic textual analysis, the maxim had lost all authority to become an object of purely literary interest.
\end{abstract}

Résumé: La sentence de saint Jérôme affirmant que "le moine n'a pas pour fonction d'instruire, mais de pleurer" occupe une place considérable dans la controverse sur la prédication et l'enseignement public des moines. Diffusée en Toscane à partir de la fin du XI siècle, elle présente l'avantage d'être l'œuvre d'un moine dont l'autorité ne pouvait pas être contestée par les défenseurs de l'ordre monastique. Issue des milieux réformateurs séculiers, elle se répand en Italie, puis en France, grâce aux collections canoniques. Popularisée par Yves de Chartres, elle est adoptée par les théologiens, puis utilisée dans les débats ecclésiologiques du début du XII siècle. Mais, vers le milieu du siècle, la critique textuelle à laquelle la soumettent les auteurs scolastiques fait perdre toute autorité à la formule de Jérôme, qui devient désormais un topos littéraire.

Les $\mathrm{XI}^{\mathrm{e}}$ et $\mathrm{XII}^{\mathrm{e}}$ siècles connaissent une transformation radicale de la place jusqu'alors réservée aux moines dans l'Église. Sous l'effet de la réforme grégorienne, les évêques revendiquent l'administration de toutes les paroisses de leurs diocèses, tandis que les chanoines réguliers développent leur mission pastorale en revenant aux traditions primitives de l'ordre canonial. Ces volontés, parfois contradictoires, s'accordent en tout cas pour contester aux moines, alors assimilés à des laïcs, la possession de biens ecclésiastiques et l'exercice du ministère'. Le

\footnotetext{
${ }^{1}$ Parmi les nombreuses études ayant traité de la controverse sur le sacerdoce des moines, on peut citer: U. Berlière, "L'exercice du ministère paroissial par les moines...», Revue bénédictine, 39, 1927, p. 227-250 et p. 340-364 ; Ph. Hofmeister, «Mönchtum und Seelsorge bis zum 13. Jahrhundert», Studien und Mitteilungen zur Geschichte des Benediktiner-Ordens und seiner Zweige, 65, 1953-54, p. 209-273; Ch. Dereine, «Le problème de la cura animarum chez Gratien », Studia Gratiana, t. II, Bologna, 1954, p. 305-318; R. Foreville, J. Leclercq, «Un débat sur le sacerdoce des moines au XII ${ }^{\mathrm{e}}$ siècle », Analecta monastica, $4^{\mathrm{e}}$ sér., Roma (Studia Anselmiana, 41), 1957, p. 8-118 ; M. Peuchmaurd, «Le prêtre ministre de la parole dans la théologie du XII ${ }^{e}$ siècle (canonistes, moines et chanoines) », Recherches de théologie ancienne et médiévale, 29, 1962, p. 52-76; J.-Fr. Lemarignier, « Le monachisme et
}

Cahiers de Recherches Médiévales, 18, 2009 
combat mené par les clercs séculiers et les chanoines réguliers était bien sûr motivé par la perception des dîmes et autres droits liés à la possession des églises paroissiales, mais il se fondait aussi sur un débat ecclésiologique portant sur la définition de l'ordre monastique et sur sa fonction propre au sein de l'Église grégorienne. Cette controverse entraina la production d'un grand nombre de textes polémiques, qui tiraient de la Bible, des canons conciliaires, des décrétales des papes et de la littérature patristique tout ce qui pouvait constituer des arguments pour condamner ou justifier le sacerdoce des moines. Parmi les autorités alléguées pour saper la position de l'ordre monastique, on retrouvait communément saint Augustin (†430), l'évêque d'Hippone, qui affirmait: "Un bon moine fait à peine un bon clerc $»^{2}$; ou saint Jérôme $(† 419 / 20)$, moine lui-même à Bethléem, qui concédait: «Les clercs nourrissent les brebis, et moi je suis nourri $»^{3}$. Mais c'est une autre sentence hiéronymienne qui attira le plus l'attention des polémistes. Dans le traité qu'il écrit en 406 contre l'Aquitain Vigilance, adversaire du culte des reliques, Jérôme défend l'utilité sociale du monachisme en définissant clairement le statut du moine et, en premier lieu, son rôle sur terre: pour lui, «le moine n'a pas pour fonction d'instruire, mais de pleurer sur lui-même ou le monde et d'attendre en tremblant la venue du Seigneur $»^{4}$. Dans l'esprit du Père de l'Église, il ne s'agissait là que de rappeler une évidence : celui qui décide volontairement de se retirer dans le désert n'a pas pour vocation d'enseigner la foi au peuple chrétiens. Mais, sept siècles plus tard, cette phrase sonnait comme un désaveu cinglant contre les prétentions des moines à exercer le ministère paroissial.

l'encadrement religieux des campagnes du royaume de France situées au nord de la Loire, de la fin $\mathrm{du} \mathrm{X}^{\mathrm{e}}$ à la fin du $\mathrm{XI}^{\mathrm{e}}$ siècle », Le istituzioni ecclesiastiche della " societas christiana» dei secoli XI-XII. Diocesi, pievi e parrochie, Milano, 1977, p. 357-394 ; C. D. Fonseca, «Monaci e canonici alla ricerca di una identità», Istituzioni monastiche e istituzioni canonicali in Occidente (1123-1215), Milano, 1980, p. 203-222; G. Constable, "Monasteries, rural churches and the cura animarum in the Early Middle Ages", Cristianizzazione ed organizzazione ecclesiastica delle campagne nell'alto Medioevo, t. I, Spoleto, 1982, p. 349-389. Sur les enjeux de la question paroissiale pour les chanoines réguliers, voir désormais $\mathrm{P}$. Montaubin, «Les chanoines réguliers et le service pastoral $\left(\mathrm{XI}^{\mathrm{e}}\right.$ $\mathrm{XIII}{ }^{\mathrm{e}}$ siècle) », Entre moines et chanoines : les chanoines réguliers. Colloque international du CERCOR, Le Puy, 29 juin-1 $1^{\text {er }}$ juillet 2006, sous presse. Nous remercions l'auteur de nous avoir communiqué le texte inédit de son article.

2 Augustinus, Ep. LX, 1: Bonus monachus vix bonum clericum faciat (éd. A. Goldbacher, Wien-Leipzig [CSEL, 34/2], 1898, p. 221, 1. 22 - p. 222, 1. 1).

${ }^{3}$ Hieronymus, Ep. XIV, 8: Clerici oves pascunt, ego pascor (éd. I. Hilberg, Wien-Leipzig [CSEL, 54], 1910, p. 55, 1. 8-9).

${ }^{4}$ Hieronymus, Adversus Vigilantium, 15 : Monachus autem non doctoris habet, sed plangentis officium, qui vel se vel mundum lugeat et Domini pavidus praestoletur adventum (S. Hieronymi presbyteri opera, t. III/5, Adversus Vigilantium, éd. J.-L. Feiertag, Turnhout [CCSL, 79C], 2005, p. 28, 1. 14-16). Le traité de Jérôme sera désormais abrégé $A d v$. Vigil., tandis que, pour l'édition de référence, on renverra dorénavant à CCSL, 79C.

${ }^{5} \mathrm{C}$ 'est d'ailleurs une réponse directe à l'attaque de Vigilance, qui demandait: Si omnes se reclauserint et fuerint in solitudine, quis celebrabit ecclesias, quis saeculares homines lucrifaciet, quis peccantes ad virtutes poterit cohortari? (CCSL, 79C, p. 28, 1. 3-5). 
Cette sentence rencontra un tel succès qu'elle accéda au rang de proverbe ${ }^{6}$. Elle figure dans une multitude d'œuvres postérieures au XII ${ }^{\mathrm{e}}$ siècle, de saint Thomas d'Aquin à l'Encyclopédie de Diderot et d'Alembert'. Elle est, bien entendu, connue des historiens de la controverse sur le sacerdoce des moines ${ }^{8}$. Mais elle l'est aussi de ceux qui ont travaillé sur l'enseignement monastique au XII ${ }^{\mathrm{e}}$ siècle $^{9}$. En effet, cette maxime avait la particularité de fonder l'incapacité des moines à enseigner, non sur leur ignorance, mais sur leur statut propre dans l'Église, ce qui définissait $a$ contrario le clerc comme détenteur par nature de la fonction d'enseigner. Surtout, elle faisait de cet officium doctoris un discriminant entre l'ordre clérical et l'ordre monastique, ce qui posait la question des enjeux ecclésiologiques de l'enseignement. Or, malgré tout l'intérêt que représente cette sentence, on ne sait rien du contexte précis dans lequel elle apparaît, ni de la manière dont elle se diffuse à la charnière des $\mathrm{XI}^{\mathrm{e}}$ et $\mathrm{XII}^{\mathrm{e}}$ siècles. Une telle recherche est désormais facilitée par l'existence de nouveaux instruments lexicographiques ${ }^{10}$. L'ensemble des fragments de la sentence de Jérôme ont ainsi été répartis en sept catégories, classées par explicit (Annexe 1). $\mathrm{Au}$ terme de ces investigations, la maxime a été retrouvée dans au moins trentequatre œuvres issues de vingt-cinq auteurs différents (Annexe 2) ${ }^{11}$. L'analyse de ce corpus se fera en deux temps: à l'examen du contexte qui voit apparaître la

${ }^{6}$ H. Walther, Proverbia sententiaeque latinitatis medii aevi, t. II, Göttingen, 1964, p. 915, $\mathrm{n}^{\circ} 15028 \mathrm{a}$, d'après le ms. Zürich 1128 , fol. $156^{\mathrm{V}}\left(\mathrm{XV}^{\mathrm{e}}\right.$ siècle).

${ }^{7}$ Elle est notamment citée dans la Pharetra pseudo-bonaventurienne (av. 1261), dans trois œuvres de Thomas d'Aquin (Summa theologiae, $\mathrm{II}^{\mathrm{a}} \mathrm{II}^{\mathrm{ae}}$, q. 187, 1, 1; De perfectione spiritualis vitae, 26; Contra impugnantes, II, 1, 2), dans le Manipulus florum de Thomas d'Irlande (vers 1306) et dans l'article «Moine » de l'Encyclopédie (1751-1772, t. X, p. 616).

${ }^{8}$ C'est notamment le cas d'U. Berlière, «L'exercice du ministère », art. cit., n. 1, p. 247 ; Ch. Dereine, «Le problème de la cura animarum », art. cit., n. 1, p. 311 ; et C. D. Fonseca, «Monaci e canonici », art. cit., n. 1, p. 210, 219.

${ }^{9}$ L'étude de référence reste celle de Ph. Delhaye, «L'organisation scolaire au XII ${ }^{\mathrm{e}}$ siècle », Traditio, 5, 1947, p. 211-268, rééd. dans Id., Enseignement et morale au XII siècle, FribourgParis, 1988, p. 1-58. On trouve de nouveaux développements sur la question dans J. Leclercq, «Les études dans les monastères du $\mathrm{X}^{\mathrm{e}}$ au $\mathrm{XII}^{\mathrm{e}}$ siècle », Los Monjes y los estudios. IV semana de estudios monasticos, Poblet 1961, Poblet, 1963, p. 105-117 ; P. Johanek, « Klosterstudien im 12. Jahrhundert», Schulen und Studium im sozialen Wandel des hohen und späten Mittelalters, éd. J. Fried, Sigmaringen, 1986, p. 35-68 ; J. Ehlers, «Dom- und Klosterschulen in Deutschland und Frankreich im 10. und 11. Jahrhundert», Schule und Schüler im Mittelalter, éd. M. Kintzinger, S. Lorenz, M. Walter, Köln, 1996, p. 29-52. Le catalogue de bibliothèque de Gorze, qui servait de base à un enseignement monastique, a en outre été analysé et édité par A. Wagner, Gorze au XI siècle. Contribution à l'histoire du monachisme bénédictin dans l'Empire, Turnhout, 1996, p. 101-190.

${ }^{10}$ Nous avons interrogé les CD-Rom suivants : Patrologia Latina Database (1997); Library of Latin Texts (CLCLT-6, 2005); Monumenta Germaniae Historica (eMGH-5, 2006); L. Fowler-Magerl, Clavis Canonum (2005); In Principio (2003); Thesaurus diplomaticus (version 1, 1997).

${ }^{11}$ Les simples réminiscences, qui ne restituent pas un fragment significatif de la sentence, ne figurent pas dans l'Annexe 2. 
sentence, dans la seconde moitié du $\mathrm{XI}^{\mathrm{e}}$ siècle, succèdera l'étude de sa diffusion, dans la première moitié du XII ${ }^{\mathrm{e}}$ siècle.

\section{L'apparition de la sentence dans la seconde moitié duXI siècle}

\section{Les premiers témoins de la sentence}

Le passage de l'Adversus Vigilantium contenant la sentence de Jérôme ne semble pas avoir particulièrement attiré l'attention des auteurs du haut Moyen Âge. Seul Raban Maur ( $†$ 856) en cite un large extrait dans son De oblatione puerorum $(\text { vers } 829)^{12}$. Ce petit traité répondait à la polémique née entre Raban Maur et son élève Gottschalk, qui prétendait se délier des vœux prononcés comme oblat de l'abbaye de Fulda ${ }^{13}$. Ayant opposé à Gottschalk un grand nombre de citations bibliques et patristiques défendant l'oblation des enfants, Raban Maur termine sa démonstration en définissant le monachisme, et donc l'oblation monastique, comme une institution divine à laquelle les lois humaines ne peuvent déroger. C'est à cette occasion qu'il utilise le traité de Jérôme contre Vigilance. Pour autant, Raban Maur ne paraît pas constituer un relais significatif de la transmission de la sentence ${ }^{14}$. Loin de la mettre en exergue, il noie la maxime dans une citation beaucoup plus longue, qui commence au milieu du chapitre précédent. Cette œuvre, peu diffusée, n'est par ailleurs conservée que dans des manuscrits autrichiens et bavarois postérieurs au $\mathrm{XI}^{\mathrm{e}}$ siècle $^{15}$. En fait, c'est en Toscane que cet extrait prend véritablement le statut de sentence.

${ }^{12}$ Adv. Vigil., 14, 1. 20 quod autem adserit-16, 1.15 mordeat (PL 107, col. 438C-440A).

${ }^{13}$ L'affaire avait été tranchée en faveur de Gottschalk par le synode de Mayence (829), mais Raban Maur, qui en refusait le verdict, rédigea ce traité peu de temps après pour soutenir sa cause. Il l'aurait même envoyé à Louis le Pieux, si l'on en croit les Miracula sanctorum in Fuldenses ecclesias translatorum (c. 15), écrits par Rudolf von Fulda ( $† 865)$ vers $842-847$ (éd. G. Waitz, Hannover [MGH, SS, 15/1], 1887, p. 340-341). Sur ce texte et la polémique qui lui est liée, voir E. Freise, «Studien zum Einzugsbereich der Klostergemeinschaft Fulda», Die Klostergemeinschaft von Fulda im früheren Mittelalter, éd. K. Schmid, t. II-III, München, 1978, p. 1003-1269, en particulier p. 1021-1229; et, en dernier lieu, M. De Jong, In Samuel's Image. Child Oblation in the Early Medieval West, Leiden-New York-Köln, 1996, p. 77-86, 160-163. Nous n'avons pas pu lire l'étude de S. Patzold, «Hraban, Gottschalk und der Traktat "De oblatione puerorum" ", Hraban Maur (v. 780-856) et son temps, Congrès international de Villeneuve d'Ascq-Amiens, 5-8 juillet 2006, à paraître.

${ }^{14}$ Les deux florilèges tardifs, qui présentent le même incipit que l'extrait cité par Raban Maur, ne semblent d'ailleurs pas appartenir à la même tradition : Troyes, BM 215, fol. $16^{\mathrm{V}}-17^{\mathrm{r}}\left(\mathrm{XII}^{\mathrm{e}}\right.$ $\mathrm{XIII}^{\mathrm{e}}$ s., Clairvaux), et Saint-Omer, BM 8, fol. $118^{\mathrm{r}}$ (XIII' ${ }^{\mathrm{e}}$ s., Clairmarais), citent en fait l' $A d v$. Vigil., 14, 1.20 quod autem adserit - 16, 1.23 voluntas (CCSL, 79C, p. LXXIV, LXXVI).

15 On ne conserve que 5 mss. de cette œuvre: Göttweig, Stiftsbibl. 54 (58), fol. $1^{\mathrm{r}}-11^{\mathrm{v}}$ (XII ${ }^{\mathrm{e}}$ s.) ; Admont, Stiftsbibl. 209, fol. $26^{\mathrm{v}}-32^{\mathrm{r}}\left(\mathrm{XV}^{\mathrm{e}} \mathrm{s}\right.$ ) ; München Clm 4783, fol. 58 $8^{\mathrm{r}}-74^{\mathrm{r}}$ $\left(X^{\mathrm{e}}\right.$ s., Benediktbeuren); Clm 18518, fol. $24^{\mathrm{r}}-36^{\mathrm{r}}$ (XV $\mathrm{XV}^{\mathrm{e}}$ s., Tegernsee) ; Oxford, Bodleian, Lyell 60 , fol. $20^{\mathrm{v}}-33^{\mathrm{v}}\left(\mathrm{XV}^{\mathrm{e}}\right.$ s., Melk). Il semble toutefois que ce traité se soit aussi trouvé à l'abbaye de Stavelot (Rabanus super regulam) en 1105 et dans celle de Lobbes (Expositio 
La première œuvre qui met en lumière la maxime de Jérôme est la collection canonique dite «en 183 titres", qui cite le fragment 3 de la sentence ${ }^{16}$. Cette compilation anonyme fut réalisée entre 1063 et 1083/5 dans le nord de la Toscane, peut-être à Lucques, par un partisan de la réforme grégorienne ${ }^{17}$. Elle inspira, à son tour, une autre collection canonique, celle dite « en 5 livres » du ms. Vat. lat. 1348, sans doute aussi rédigée en Toscane vers 1083-1085 ${ }^{18}$. Simple abrégé de la Collection en 183 titres, cette dernière compilation cite naturellement l'extrait sous une forme identique ${ }^{19}$. C'est peut-être à l'une ou l'autre de ces collections italiennes que l'on doit de retrouver la sentence de Jérôme en annexe d'une version tardive de la Collectio Sinemuriensis, l'une des premières collections réformatrices du clergé français $^{20}$. Le fragment 3 est en effet cité dans l'appendice du ms. Orléans, BM 306, mais il pourrait tout aussi bien s'agir d'un emprunt commun à un même florilège

Rabani super regulam Benedicti) au XV $\mathrm{XV}^{\mathrm{e}} \mathrm{s}$. (R. Kottje, «Hrabanus Maurus », Die deutsche Literatur des Mittelalters. Verfasserlexikon, t. IV, Berlin-New York, 1982-83, col. 192).

${ }^{16}$ Collectio CLXXXIII titulorum, 143, 5: De officio monastico. JERONIMUS IN EPISTOLA ADVERSUS VigILANTIUM. Monachus non doctoris habet, sed plangentis officium, qui vel se vel mundum lugeat et Domini pavidus prestoletur adventum (Liber canonum diversorum sanctorum patrum sive Collectio in CLXXXIII titulos digesta, éd. G. Motta, Città del Vaticano, 1988, p. 228).

${ }^{17}$ L. Kéry, Canonical Collections of the Early Middle Ages (ca. 400-1140). A Bibliographical Guide to the Manuscripts and Literature, Washington, 1999, p. 216-217 ; L. Fowler-Magerl, Clavis Canonum. Selected Canon Law Collections before 1140, Hannover, 2005, p. 100-102. Le plus récent canon cité est une lettre d'Alexandre II (JL 4500), datée de 1063 (Liber canonum, op. cit., n. 16, p. XXVI). Le ms. de Santa Maria Novella (Firenze, Bibl. Naz., Conv. soppr., A.IV.269) était sans doute originaire de Lucques : copié vers 1120, il fut très tôt conservé à Prato, où les dominicains de Florence l'acquirent à la fin du XIII ${ }^{\mathrm{e}}$ ou au début du $\mathrm{XIV}^{\mathrm{e}}$ s. (P. Fournier, G. Le Bras, Histoire des collections canoniques en Occident depuis les Fausses Décrétales jusqu'au Décret de Gratien, t. II, Paris, 1932, p. 151-152).

${ }_{18}$ L. Kéry, Canonical Collections, op. cit., n. 17, p. 217-218; L. Fowler-Magerl, Clavis Canonum, op. cit., n. 17, p. 102-104. Cette collection fut peut-être composée à Florence, dans la mesure où son unique témoin appartenait, dès le Moyen Âge, au monastère florentin de Santa Maria dei Angeli.

${ }^{19}$ Collectio Vlibrorum, 3, 28, 3 : Jeronimus in EPIStola adVERSUS Vigilantium. Monachus non doctoris [...] adventum (V. Wolf von Glanvell, «Die Canonessammlung des Cod. Vatican. lat. $1348 »$, Sitzungsberichte der kaiserlichen Akademie der Wissenschaften in Wien. Philos.-hist. Klasse, 136/2, 1897, p. 37).

${ }^{20}$ Le ms. Orléans, BM 306 (fin XI ${ }^{\mathrm{e}}$-déb. XII ${ }^{\mathrm{e}}$ s., Fleury), contient en fait une importante annexe, composée notamment d'extraits des collections d'Anselme de Lucques et de Deusdedit. L'auteur de cet appendice à la Collectio Sinemuriensis a sans doute eu accès à une compilation italienne qui utilisait ces deux sources (L. Fowler-Magerl, Clavis Canonum, op. cit., n. 17, p. 108). Sur l'origine et l'importance de cette collection canonique, voir L. Fowler-Magerl, "Vier französische und spanische vorgratianische Kanonessammlungen », Aspekte europäischer Rechtsgeschichte. Festgabe für Helmut Coing zum 70. Geburtstag, Frankfurt am Main, 1982, p. 123-146. 
hiéronymien ${ }^{21}$. À peu près à la même époque, on rencontre le passage de l'Adversus Vigilantium dans l'un des remaniements de la collection canonique d'Anselme de Lucques $(\dagger 1086)$ : la version dite «A Aucta $»^{22}$. La généalogie des multiples recensions de la collection de cet évêque réformateur (A, A', A Aucta, Bb, B et C) a fait l'objet de nombreux débats entre spécialistes et a produit, depuis le XIX ${ }^{\mathrm{e}}$ siècle, une abondante littérature, qui ne résout pas toutes les questions d'attribution ${ }^{23}$. L'historiographie actuelle tend néanmoins à considérer que le remaniement de la collection originale, d'où sont issues les versions $\mathrm{A}$ Aucta et $\mathrm{B}$, aurait été réalisé au monastère San Benedetto de Polirone, près de Mantoue, du vivant même d'Anselme de Lucques $^{24}$. Or, cette version A Aucta, qui daterait sans doute de la fin des années 1080, contient le fragment 6 de la sentence, soit une forme sensiblement plus longue que celle de la Collection en 183 titres $^{25}$. On retrouve enfin le même fragment, avec quelques variantes, dans un recueil peut-être d'origine poitevine, la Collection en

\footnotetext{
${ }^{21}$ Collectio Sinemuriensis, App. 680 : In EAdem [Hieronimus in EPISTOLA]. Monachus non doctoris habet, sed plangentis [...] pavidus prestoletur adventum (Orléans, Bibl. mun., 306, p. 321, d'après L. Fowler-Magerl). Sur les 21 fragments cités entre les c. 669 et 689 de cet appendice, on ne trouve pas moins de dix-huit citations de Jérôme, dont un autre extrait de l'Adv. Vigil. (14, 1. 16-19 in sanctis - spiritualia) au c. 679, qui précède immédiatement la sentence. $\mathrm{Si}$, comme on peut le penser, ce bloc de sources a été tiré d'un même florilège hiéronymien, que nous ne sommes pas parvenu à identifier, il est intéressant de noter que le fragment 3 de la sentence y était, semble-t-il, déjà individualisé.

${ }^{22}$ Cette version de la collection d'Anselme de Lucques avait été baptisée «A Ven» par Fr. Thaner, qui l'avait reconnue dans le ms. Venezia, Marciana, lat. IV.55 (XII ${ }^{\mathrm{e}}$ s., région de Padoue ?). Mais, P. Landau ayant découvert une seconde copie de la même version dans le ms. Mantova, BC, C.II.23 (déb. XII ${ }^{\mathrm{e}}$ s., San Benedetto di Polirone), elle fut rebaptisée «A Aucta » par G. Motta, «La redazione A "Aucta" della Collectio Anselmi episcopi Lucensis », Studia in honorem eminentissimi cardinalis Alphonsi M. Stickler, éd. R. J. Castillo Lara, Roma, 1992, p. 375-449.

${ }^{23}$ Pour un inventaire détaillé de la bibliographie sur la question, voir L. Kéry, Canonical Collections, op. cit., n. 17, p. 221-226; et les compléments de L. Fowler-Magerl, Clavis Canonum, op. cit., n. 17, p. 144-145, 148, 221, 227.

${ }^{24}$ L. Fowler-Magerl, Clavis Canonum, op. cit., n. 17, p. 147. Il n'est pas inutile de rappeler que, bien qu'étroitement liée à la version A Aucta, la version B ne contient pas la sentence de Jérôme.

${ }^{25}$ Anselmus Lucensis, Collectio canonum (version A Aucta), 7, 128: Quod monachus non doctoris sed plangentis habet officium. JERONIMUS. Monachus non doctoris habet, sed plangentis officium, quod se vel mundum lugeat et Domini pavidus prestoletur adventum, qui sciens inbecillitatem suam et vas fragile quod portat, timet offendere. Unde et mulierum maxime adholescentularum vitet aspectum, ne eum capiat oculus meretricis, ne forma pulcherrima ad illicitos ducat amplexus (Venezia, Bibl. Naz. Marciana, lat. IV.55, fol. 166 ${ }^{\mathrm{r}}$ ). Nous tenons à remercier notre collègue, Linda Fowler-Magerl, de nous avoir permis d'obtenir la copie du ms. de Venise. On peut ainsi constater que le canon de la collection anselmienne comporte deux lacunes par rapport au texte original : la première de 6 mots (Adv. Vigil., 15, 1.18 offendere [ne impingat-frangatur] unde); la seconde de 34 (Adv. Vigil., 15, 1. 19 aspectum [et in tantum - 16, 1. 3 patiar] ne).
} 
13 livres du ms. Berlin, Savigny 3, compilée vers 1089, qui aurait utilisé la version A Aucta d'Anselme de Lucques, comme l'a montré Peter Landau ${ }^{26}$.

En dehors des collections canoniques, la sentence est citée, dans un tout autre contexte, mais dans la même région, par un traité écrit en faveur de l'empereur Henri IV et attribué à un certain Petrus Crassus : la Defensio Heinrici $\mathrm{IV} \mathrm{regis}^{27}$. Si la datation du texte semble bien établie (1084) $)^{28}$, l'identité de son auteur est loin d'être définie. Pour reprendre l'expression d'Ennio Cortese, Petrus Crassus est un personnage dont rien n'est certain, pas même le nom ${ }^{29}$. Le fait est que la dédicace qui termine la Defensio laisse entendre que «Petrus » et «Crassus » ne seraient pas une seule et même personne ${ }^{30}$. Mais, à la suite de Ficker, qui croyait avoir reconnu l'hypothétique auteur dans le «Petrus Grasso » d'un diplôme de Ravenne (1074), l'historiographie a longtemps écarté les doutes qui pesaient sur l'association de ces deux noms ${ }^{31}$. Si l'origine précise et la fonction exacte du rédacteur demeurent inconnues, il paraît en revanche probable que l'œuvre fut produite dans l'entourage de l'archevêque Ghiberto de Ravenne, alors antipape sous le nom de Clément III $(1080-1100)^{32}$. La Defensio cite la maxime sous la forme du fragment 5, qui n'est ni

\footnotetext{
${ }^{26}$ Collectio XIII librorum, 6, 94 : Jeronimus. Ne monachus doceat. Monachus non doctoris, sed lugentis habet [...] illicitos ducat amplexus (Berlin, Staatsbibl. Preußischer Kulturbesitz, Savigny 3 , fol. $83^{\text {rb }}$, d'après L. Fowler-Magerl). Sur la date et l'origine de cette collection, voir P. Fournier, G. Le Bras, Histoire des collections, op. cit., n. 17, t. II, p. 258 ; L. FowlerMagerl, Clavis Canonum, op. cit., n. 17, p. 155-156. Sur les relations entre cette collection et celle d'Anselme de Lucques, voir P. Landau, «Erweiterte Fassungen der Kanonessammlung des Anselm von Lucca aus dem 12. Jahrhundert», Sant'Anselmo, Mantova e la lotta per le investiture, éd. P. Golinelli, Bologna, 1987, p. 329-330, rééd. dans Id., Kanones und Dekretalen, Goldbach (Bibliotheca eruditorum, 2), 1997, p. 87*-88*.

${ }^{27}$ Éd. L. von Heinemann, Hannover (MGH, L. d. l., 1), 1891, p. 432-453. Ce traité n'est conservé que dans une copie très défectueuse du XVI ${ }^{\mathrm{e}}$ s. (Hannover, Landesbibl., XI671, fol. $65^{\mathrm{r}}-89^{\mathrm{v}}$ ). Il a été édité une première fois par H. Sudendorf (Registrum oder merkwürdige Urkunden für die deutsche Geschichte, t. I, Jena, 1849, p. 22-50, n 13-14), qui a proposé un certain nombre de corrections, reprises ensuite par J. Ficker (Forschungen zur Reichs- und Rechtsgeschichte Italiens, t. IV, Innsbruck, 1874, p. 106-124, $\mathrm{n}^{\circ} 80$ ).

${ }^{28}$ Ce libelle anti-pontifical aurait été publié après la prise de Rome par les armées impériales en mars 1084, à l'occasion du synode réuni par Henri IV pour déposer Grégoire VII (éd. cit., n. 27, p. 432).

${ }^{29}$ E. Cortese, Il diritto nella storia medievale, t. I, Roma, 1995, p. 357.

${ }^{30} \mathrm{Si}$ les vers n'ont pas été interpolés, ce serait un certain Petrus qui aurait demandé à Crassus de rédiger ce traité : Henrice rex amabilis, / Qui Romae victor existis, / Hunc librum nostrum accipis, / Quem vestri Crassus tradidit / Exemplis patrum editum / Rogatu Petri conditum (éd. cit., n. 27, p. 453).

31 J. Ficker, Forschungen zur Reichs- und Rechtsgeschichte Italiens, op. cit., n. 27, t. III, p.112-114. Cette association a été récemment contestée par I. Heidrich, Ravenna unter Erzbischof Wibert (1073-1100). Untersuchungen zur Stellung des Erzbischofs und Gegenpapstes Clemens III. in seiner Metropole, Sigmaringen, 1984, p. 152.

${ }^{32}$ E. Cortese, Il diritto nella storia medievale, op. cit., n. 29, t. I, p. 358.
} 
celle de la Collection en 183 titres, ni celle de la version A Aucta d'Anselme de Lucques $^{33}$.

Ainsi, les trois premiers témoins de la sentence la révèlent sous trois aspects différents. Compte tenu de leur taille, il est impossible que les fragments de la Defensio et de la version A Aucta d'Anselme de Lucques proviennent de la Collection en 183 titres. Si l'on peut toujours supposer une influence de la plus ancienne compilation sur les deux autres, il paraît toutefois évident que ces dernières ont recopié leurs citations ailleurs, puisqu'elles contiennent des extraits plus longs de 1'Adversus Vigilantium ${ }^{34}$. De même, le fragment 5 de la Defensio ne peut, en aucun cas, avoir été tiré du fragment 6 de la version A Aucta, car les deux passages présentent des lacunes incompatibles ${ }^{35}$. Il faut donc admettre que ces trois premiers témoins ont extrait la maxime de manière indépendante. Cela invite à s'interroger sur la connaissance de l'œuvre de saint Jérôme en Italie à la fin du $\mathrm{XI}^{\mathrm{e}}$ siècle. Les premiers compilateurs qui révèlent la sentence ont eux-mêmes de cette œuvre une connaissance très variable. La Defensio Heinrici IV regis fait un usage rare, et parfois fautif, des citations hiéronymiennes ${ }^{36}$. À l'inverse, l'auteur de la Collection en 183 titres connaît intimement les écrits du Père de l'Église: les trois quarts des canons attribués à Jérôme ne proviennent apparemment d'aucune autre collection canonique $^{37}$. On sait que l'Adversus Vigilantium a circulé très tôt au sein des recueils

\footnotetext{
${ }^{33}$ Petrus Crassus, Defensio Heinrici IV regis, 5 : Monachus, inquit, non doctoris habet, sed plangentis officium, qui vel se vel mundum lugeat et Domini pavidus prestoletur adventum, qui sciens imbecillitatem suam et vas fragile quod portat, timeat offendere, ne impingat et corruat atque frangatur. Et in tantum castigator sui sit, ut etiam quae tuta sunt pertimescat (éd. cit., n. 27, p. 442, 1.3-7). On peut noter que l'extrait de la Defensio comporte une lacune de 7 mots par rapport au texte original (Adv. Vigil., 15, 1. 18-19 frangatur [unde et mulierumaspectum] et in).

${ }^{34}$ On sait, par exemple, qu'Anselme de Lucques fut certainement orienté dans ses choix par la lecture de la Collection en 183 titres, mais qu'il ne l'utilisa pas directement, puisqu'il cite des passages plus longs pour certains canons communs (L. Fowler-Magerl, Clavis Canonum, op. cit., n. 17, p. 100-101). Il en va peut-être de même pour l'auteur de la version A Aucta.

${ }^{35}$ Cf. supra, n. 25 et 33.

${ }^{36}$ En dehors de l'extrait de l'Adv. Vigil., l'auteur de la Defensio n'allègue Jérôme que dans deux autres cas. Or, l'une de ces citations (éd. cit., n. 27, p. 440, 1. 5-7) provient en fait d'un passage de l'Epistola ad fratres Lugdunenses d'Alcuin (PL 100, col.293A), repris mot pour mot par Jessé d'Amiens dans son Epistola de baptismo (PL 105, col. 792B) et remanié par Hincmar de Reims dans son De una et non trina deitate (PL 125, col. 501C).

${ }^{37}$ Sur les 19 canons attribués à Jérôme, on en compte un tiré du Décret de Burchard de Worms (183T, 164, $4<$ Decretum, 12, 16), deux peut-être repris de collections antérieures (183T, 93, $10=3^{a}$ collectio Ambrosiana 46 inf., 28g; 183T, 168, $4=$ Collectio 5 librorum [Vat. lat. 1339], 4, 383a), un autre que l'on retrouve dans des collections contemporaines (183T, 91, $1=$ Collectio Barberiniana, 11, 3 = Collectio Sinemuriensis [Semur, BM 13], 3, 97), une seule fausse attribution $(183 \mathrm{~T}, 56,2)$ et surtout 14 canons inédits $(183 \mathrm{~T}, 75,9$ et $19 ; 91,6$; $93,3 ; 97,7 ; 143,4$ à $6 ; 147,77 ; 150,4 ; 152,4$ et $12 ; 164,31 ; 172,1)$. Le canon faussement attribué à Jérôme se retrouve, dès 1078, dans une lettre des clercs de Noyon à ceux de Cambrai (éd. H. Boehmer, Hannover [MGH, L. d. l., 3], 1897, p. 576, 1. 38 - p. 577, 1.9) et il est recopié, à la fin du $\mathrm{XI}^{\mathrm{e}}$ s., dans la Collectio XX $\operatorname{Xibrorum}(2,27) \mathrm{du}$ ms. Vat.
} 
épistolaires de saint Jérôme. Il figure notamment, dès le $\mathrm{X}^{\mathrm{e}}$ siècle, dans la collection dite « des 123 pièces », qui mêle lettres et traités hiéronymiens ${ }^{38}$. Il est toutefois plus vraisemblable d'imaginer qu'un extrait significatif de l'Adversus Vigilantium, commençant par l'incipit « Monachus non doctoris... », avait déjà été isolé dans l'un des florilèges utilisés par ces compilateurs ${ }^{39}$. Malheureusement, on manque d'études sur les florilèges patristiques des $\mathrm{X}^{\mathrm{e}}$ et $\mathrm{XI}^{\mathrm{e}}$ siècles ${ }^{40}$. Le dépouillement des recueils édités n'a pas permis de repérer un tel extrait dans ceux du haut Moyen Âge, et la présence de la sentence dans les florilèges - mieux connus - du XII ${ }^{\mathrm{e}}$ siècle ne suffit pas à prouver qu'elle ait circulé de manière individualisée au siècle précédent ${ }^{41}$. Ainsi, dans l'état actuel de nos connaissances, il n'est pas possible de préciser davantage les sources formelles des trois premiers témoins de la maxime.

Il semble donc que cet extrait de l'Adversus Vigilantium n'ait véritablement été élevé au rang de sentence qu'à la fin du $\mathrm{XI}^{\mathrm{e}}$ siècle, en Italie centroseptentrionale, et plus précisément dans un espace compris entre Lucques, Mantoue et Ravenne. Or, on sait qu'entre 1055 et 1070, l'Ombrie et la Toscane connaissaient

lat. 1350, sous l'inscription Ex decretis Jeronimi. La Collection en 183 titres contient, en outre, trois extraits hiéronymiens non identifiés par l'auteur : l'un est attribué à saint Augustin dès Burchard de Worms (183T, 87, $8<$ Decretum, 11, 15); les deux autres ne sont pas attribués (183T, 166, $5<$ Decretum, 11, 55 ; 183T, 178, 3).

${ }^{38}$ Il s'agit d'une collection bien connue dont les plus anciens témoins sont les mss. Padova, Antoniana, 73 scaff. IV ( $\mathrm{X}^{\mathrm{e}}$ s.), Paris lat. 1871 ( $\mathrm{X}^{\mathrm{e}}$ s.), Vat. lat. $354\left(\mathrm{XI}^{\mathrm{e}} \mathrm{s}\right.$.) et Vat. lat. 355-356 $\left(\mathrm{X}^{\mathrm{e}} \mathrm{s}\right.$.). Sur cette collection et ses remaniements ultérieurs, voir P. Lardet, "Épistolaires médiévaux de S. Jérôme : jalons pour un classement ", Freiburger Zeitschrift für Philosophie und Theologie, 28, 1981, p. 271-289.

${ }^{39} \mathrm{C}$ 'est peut-être par le biais d'un florilège hiéronymien que la maxime a été insérée dans l'appendice du ms. Orléans, BM 306 (cf. supra, n. 21).

40 Au-delà des synthèses d'H. Rochais («Florilèges latins», Dictionnaire de spiritualité ascétique et mystique, t. V, Paris, 1964, col. 435-460) et de M. A. et R. H. Rouse («Florilegia of Patristic Texts ", Les genres littéraires dans les sources théologiques et philosophiques médiévales, Louvain-la-Neuve, 1982, p. 165-180), on peut citer quelques travaux sur des florilèges hiéronymiens : J. Leclercq, «Saint Jérôme docteur de l'ascèse, d'après un centon monastique », Revue d'ascétisme et de mystique, 25, 1949, p. 140-145 ; R. Étaix, "Un ancien florilège hiéronymien », Sacris Erudiri, 21, 1972-73, p. 5-34 ; P.-I. Fransen, «Description de la collection hiéronymienne de Florus de Lyon sur l'Apôtre ", Revue bénédictine, 94, 1984, p. 195-228 ; J.-L. Feiertag, «Vigilance et Jérôme sur la lecture d'Origène dans un florilège du $\mathrm{XI}^{\mathrm{e}}$ siècle », Revue d'études augustiniennes et patristiques, 51, 2005, p. 279-296.

${ }^{41}$ On a pu reconnaître de tels extraits dans 5 florilèges du XII ${ }^{\mathrm{e}} \mathrm{s}$. : Bern, Bürgerbibl., 460, fol. $52^{\text {ra }}-52^{\text {va }}$ (Adv. Vigil., 15, 1. 14 monachus - 16, 1. 18 colloquia) [Fl. Nuvolone, «Notulae Manuscriptae (III et IV) », Freiburger Zeitschrift für Philosophie und Theologie, 26, 1979, p. 550]; Bologna, Bibl. Univ., 2535, fol. $141^{\mathrm{v}}$ (Adv. Vigil., 15, 1. 14 monachus - 16, 1. 20 non habet) [C. D. Fonseca, Medioevo canonicale, Milano, 1970, p. 142] ; Firenze, Bibl. Medicea Laurenziana, S. Croce, Plut. V sin. cod. 7, fol. $99^{\mathrm{v}}$ (fragment 3) ; Roma, Bibl. Angelica, 1895, fol. $16^{\mathrm{v}}$ (fragment 3) ; Sélestat, Bibl. mun., 128, fol. 44 $-45^{\mathrm{r}}$ (fragment 3) [CCSL, 79C, p. LXXII-LXXIII]. Sur les florilèges patristiques de cette époque, voir Th. Falmagne, «Les cisterciens et les nouvelles formes d'organisation des florilèges aux $12^{\mathrm{e}}$ et $13^{\mathrm{e}}$ siècles $»$, Archivum Latinitatis Medii Aevi, 55, 1997, p. 73-176. 
de fortes tensions entre moines et clercs séculiers, sous l'effet de la campagne antisimoniaque orchestrée par les disciples du réformateur Jean Gualbert ( $†$ 1073), le fondateur du monastère de Vallombreuse ${ }^{42}$. C'est en fait dans ce contexte que la maxime se distingue nettement du reste de l'œuvre en tirant profit du caractère percutant de la première phrase qui débute invariablement tous les fragments de l'extrait. C'est surtout à cette époque qu'on lui accorde une valeur probatoire dans les débats ecclésiologiques sur le statut de l'ordre monastique. Le plan des collections canoniques qui contiennent cette maxime ne laisse planer aucun doute sur le rôle qui est désormais assigné aux moines dans la nouvelle Église grégorienne: dans la Collection en 183 titres, la sentence hiéronymienne $(143,5)$, censée définir la fonction même du moine (De officio monastico), est rapprochée d'un canon du concile d'Agde interdisant la fondation d'un monastère sans autorisation épiscopale $(143,2)^{43}$, d'une lettre attribuée au pape Gélase interdisant aux moines de se mêler des affaires séculières $(143,3)^{44}$, d'une lettre de Jérôme subordonnant clairement les moines aux clercs $(143,6)^{45}$ et d'une lettre du pape Léon le Grand (440-461) interdisant aux moines de prêcher $(143,7)^{46}$. Le message est clair : l'ordre monastique est subordonné à l'ordre clérical et ne doit, en aucun cas, exercer de responsabilités sacerdotales. Ce n'était donc pas, à proprement parler, l'enseignement scolaire des moines qui était visé, mais l'usurpation par eux du ministère de la parole. Par rapport aux autres canons, l'intérêt de ce passage de l'Adversus Vigilantium résidait bien sûr dans son caractère de formule. Le compilateur de la version A Aucta d'Anselme de Lucques ne s'y est d'ailleurs pas trompé en adoptant comme rubrique une expression qui allait devenir la version classique de la maxime (Quod monachus non doctoris sed plangentis habet officium). Mais, pour les clercs réformateurs, ce texte présentait surtout l'avantage d'être placé sous l'autorité d'un des pères du monachisme, ce qui rendait autrement plus délicate sa contestation par les moines. En somme, les canonistes italiens sont parvenus à faire de cette exaltation par Jérôme de l'état monastique un véritable instrument idéologique antimonastique. Le fait que cette sentence soit reprise, dès 1084, par un adversaire acharné de Grégoire VII n'invalide en rien son origine grégorienne. Cela ne fait, au contraire, que confirmer l'autorité déjà acquise par ce texte à la fin du $\mathrm{XI}^{\mathrm{e}}$ siècle.

\section{La sentence chez Yves de Chartres}

Les premiers témoins italiens de la maxime n'ont eu, à vrai dire, qu'une importance locale. Sans même parler de la Defensio Heinrici IV regis, qui ne doit sa survie qu'à une transcription du $\mathrm{XVI}^{\mathrm{e}}$ siècle, les premières compilations canoniques

\footnotetext{
${ }^{42}$ D'abord soutenus par le cardinal Humbert et Pierre Damien, ces anti-simoniaques radicaux sont rappelés à l'ordre par Alexandre II, qui enjoint les moines à rentrer dans leurs monastères (Ch. Dereine, «Le problème de la cura animarum », art. cit., n. 1, p. 311).

${ }^{43}$ Conc. Agde (506), c. 27 (Concilia Galliae, a. 314-a. 506, éd. Ch. Munier, Turnhout [CCSL, 148], 1963, p. 205, 1. 240-241).

${ }^{44}$ Il s'agit en fait d'une pseudo-décrétale du pape Pélage II ( $\left.\mathrm{JK} \uparrow 1050\right)$.

${ }^{45}$ Hieronymus, Ep. XIV, 8 (éd. cit., n. 3, p. 55, 1. 7-p. 56, 1. 4).

${ }^{46}$ Leo Magnus, Ep. CXX, 6 (JK $496=$ PL 54, col. 1054B)
} 
qui citent ce passage furent assez peu diffusées ${ }^{47}$. Certes, ces collections italiennes étaient connues au-delà des Alpes avant le début du XII ${ }^{\mathrm{e}}$ siècle, puisqu'on retrouve leurs fragments hiéronymiens dans deux compilations françaises de cette époque : la Collection en 13 livres du ms. Berlin, Savigny3, et l'annexe de la Collectio Sinemuriensis dans le ms. Orléans, BM306. Mais ces témoins ultramontains restèrent eux-mêmes confidentiels. En fait, ce qui assura le succès définitif de la sentence de Jérôme fut son adoption par l'un des plus célèbres canonistes de l'époque, l'évêque Yves de Chartres († vers 1116).

À lui seul, Yves cite ce passage à six reprises. La première occurrence figure dans son Décret, sous la forme du fragment 7, qui est la plus longue citation de la maxime hiéronymienne ${ }^{48}$. Cette collection canonique fut rédigée à Chartres, peu après 1093, si l'on en croit la date du plus récent document datable qu'elle contient ${ }^{49}$. Yves réutilise ensuite le même canon du Décret $(7,3)$ dans les deux autres collections qu'il a écrites ou supervisées: la Panormie $(3,176)$ et la Collectio Tripartita $(3,11,2)^{50}$. La Panormie aurait été composée vers 1094 : elle est en effet postérieure au Décret, qu'elle révise et abrège, mais ne contient aucun canon du

${ }^{47}$ La Collection en 183 titres n'est transmise que par trois copies florentines (Liber canonum, op. cit., n. 16, p. XVII). Son abrégé en 5 livres ne subsiste qu'en un seul ms. conservé à Florence dès le Moyen Âge (P. Fournier, G. Le Bras, Histoire des collections, op. cit., n. 17, t. II, p. 131). Quant à la version A Aucta d'Anselme de Lucques, elle n'est représentée que par deux témoins (G. Motta, «La redazione $A$ “Aucta" », art. cit., n. 22, p. 384-387).

${ }^{48}$ Ivo Carnotensis, Decretum, 7, 3: IDEM [HIERONIMUS] IN EPISTOLA AD RIPARIUM ET DESIDERIUM PRESBITEROS. Unde supra [De monachorum singularitate]. Monachus non doctoris habet, sed plangentis officium, qui vel se vel mundum lugeat et Domini pavidus prestoletur adventum, qui sciens imbecillitatem suam et vas fragile quod portat, timet offendere, ne impingat et corruat atque frangatur. Unde et mulierum maximeque adolescentularum vitat aspectum et intantum castigator sui est, ut etiam que tuta sunt pertimescat. Cur, inquis, pergis ad eremum? Videlicet ut te non audiam, non videam, ut tuo furore non movear, ut tua bella non patiar, ne me capiat oculus meretricis, ne forma pulcerrima ad illicitos ducat amplexus. Respondebis: Hoc non est pugnare, sed fugere. Sta in acie, adversariis armatus obsiste, ut postquam viceris coroneris. Fateor imbecillitatem meam. Nolo spe pugnare victorie, ne perdam aliquando victoriam (éd. électronique de M. Brett et Br. Brasington: www.wtamu.edu/ bbrasington/). Cette édition provisoire remplace d'ores et déjà celle de Migne (PL 161, col. 543C-D).

${ }^{49}$ L. Kéry, Canonical Collections, op. cit., n. 17, p. 250. Il convient de préciser que certains auteurs penchent pour une datation plus tardive, après 1095 et même après 1099 (M. Brett, «Urban II and the collections attributed to Ivo of Chartres », Proceedings of the Eighth International Congress of Medieval Canon Law, San Diego, University of California at La Jolla, 21-27 August 1988, éd. S. Chodorow, Città del Vaticano, 1992, p. 44).

${ }^{50}$ La Panormie (PL 161, col. 1172A-B) est en cours de réédition sur le site de Br. Brasington [www.wtamu.edu/ bbrasington/]. La version actuellement disponible ne présente pas de différences significatives avec le texte du Décret $(7,3)$. La Collectio Tripartita est encore inédite pour la partie qui contient la sentence de Jérôme. Mais l'incipit, analysé d'après un ms. de la seconde recension, présente au moins une variante par rapport aux deux autres versions ivoniennes du canon : Monachus non doctoris, sed plangentis habet [...] (Paris, Bibl. nat., Lat. 3858B, fol. $160^{\text {vb }}$, d'après L. Fowler-Magerl). 
concile de Clermont (1095) $)^{51}$. Quant à la troisième partie de la Collectio Tripartita, c'est aussi un abrégé du Décret, ajouté avant la fin du $\mathrm{XI}^{\mathrm{e}}$ siècle aux deux premières parties de l'œuvre, qui avaient été rédigées ensemble dès 1093 ou $1094^{52}$. Contrairement au Décret, dont le succès fut limité, la Tripartita et surtout la Panormie furent recopiées à grande échelle ${ }^{53}$. Cette dernière fut même l'une des œuvres canoniques les plus diffusées du XII ${ }^{\mathrm{e}}$ siècle. Elle servit de source formelle à bon nombre de collections françaises et fut largement utilisée pour composer le Décret de Gratien ${ }^{54}$. À côté de cette littérature juridique, Yves de Chartres écrivit aussi une abondante correspondance, au sein de laquelle la sentence est citée trois fois : sous la forme du fragment 1, dans la Lettre $36(1094)^{55}$, et sous celle du fragment 2, dans les Lettres $41(1094)^{56}$ et 108 (vers 1101) ${ }^{57}$. L'intérêt de telles citations n'est pas à négliger, car la correspondance ivonienne pouvait aussi bien servir de source doctrinale que de modèle rhétorique, ce qui lui permettait de toucher un public plus varié que celui des collections canoniques. Ces lettres nous sont transmises par une cinquantaine de manuscrits, principalement du XII ${ }^{\mathrm{e}}$ siècle, que Jean Leclercq a classés en deux groupes, le type II étant un remaniement du type $\mathrm{I}^{58}$.

${ }^{51}$ P. Fournier, G. Le Bras, Histoire des collections, op. cit., n. 17, t. II, p. 95-97. Certains auteurs admettent toutefois que la Panormie aurait pu être écrite après 1095 : R. Sprandel, Ivo von Chartres und seine Stellung in der Kirchengeschichte, Stuttgart, 1962, p. 73, n. 61 ; M. Brett, «Urban II and the collections », art. cit., n. 49, p. 46.

${ }^{52}$ Bien que publiés à part, les deux éléments de la Collectio Tripartita semblent avoir été réunis très tôt par la tradition, puisqu'on ne conserve pratiquement aucun témoin qui les transmette séparément (P. Fournier, G. Le Bras, Histoire des collections, op. cit., n. 17, t. II, p. 66). Le seul ms. qui ne contient pas la troisième partie de l'œuvre (Berkeley, Law Libr., Robbins 102) est en fait un témoin de la seconde recension, réalisée après la réunion des deux éléments de la collection (L. Fowler-Magerl, Clavis Canonum, op. cit., n. 17, p. 188).

${ }^{53}$ À côté des 7 mss. plus ou moins complets du Décret (P. Landau, «Das Dekret des Ivo von Chartres. Die handschriftlische Überlieferung im Vergleich zum Text in den Editionen des 16. und 17. Jahrhunderts », ZSS, Kan. Abt., 70, 1984, p. 8-13, rééd. dans Id., Kanones und Dekretalen, op. cit., n. 26, p. 124*-129*), on en conserve 22 de la Tripartita et près de 150 de la Panormie (L. Kéry, Canonical Collections, op. cit., n. 17, p. 244-245, 254-258).

${ }^{54}$ P. Landau, «Neue Forschungen zu vorgratianischen Kanonessammlungen und den Quellen des gratianischen Dekrets », Ius Commune, 11, 1984, p. 25, rééd. dans Id., Kanones und Dekretalen, op. cit., n. 26, p. 201*.

${ }^{55}$ Ivo Carnotensis, Ep. XXXVI: Monachus non docentis, sed dolentis habet officium (Yves de Chartres, Correspondance, éd. et trad. J. Leclercq, t. I, Paris, 1949, p. 150). Cette lettre fait allusion à une bulle d'Urbain II, datée du 2 nov. 1091 ou 1093 (JL 5493). Elle pourrait donc avoir été écrite en 1092 ou en 1094, mais sa place dans le recueil fait plutôt pencher son éditeur pour la date de 1094 (ibid., p. 146, n. 7).

${ }^{56}$ Ivo Carnotensis, Ep. XLI: Monachus non habet officium docentis, sed plangentis, qui se et mundum lugeat (ibid., p. 164).

${ }^{57}$ Ivo Carnotensis, Ep. CVIII : Monachus non docentis habet officium, sed plangentis, qui se et mundum lugeat (PL 162, col. 126D). Cette lettre fait allusion à une bulle de Pascal II, datée du 19 nov. 1100 (JL 5847).

${ }^{58}$ J. Leclercq, «La collection des lettres d'Yves de Chartres », Revue bénédictine, 56, 194546, p. 108-125, en particulier p. 111-112. La liste des mss. doit être complétée par les 
Tout porte à croire que la première collection épistolaire a été réalisée par l'auteur lui-même à la fin de son épiscopat: la lettre la plus récente des recueils de type I date en effet de $1114^{59}$. Or, les trois lettres qui reprennent la maxime appartiennent à cette première recension.

À l'instar des compilateurs italiens, Yves de Chartres allègue le texte de Jérôme pour dénier aux moines toute responsabilité séculière. D'après le Livre VII du Décret, qui est entièrement consacré à la singularité de l'état monastique par rapport aux clercs, les moines doivent se détourner du siècle et ne se consacrer qu'à l'ascèse et à la solitude. Le long fragment hiéronymien cité au canon 3 insiste d'ailleurs plus sur la fuite du monde que sur l'interdiction d'enseigner qui débute l'extrait. Yves n'est toutefois pas insensible au caractère percutant de la formule. On le constate dans ses lettres, qui isolent des fragments plus courts, centrés sur la première phrase de la sentence. Il en va ainsi de la Lettre 36, adressée à l'évêque Pierre II de Poitiers (1087-1115) et qui constitue un véritable petit dossier patristique contre les prétentions de l'ordre monastique. Dénonçant auprès de son collègue les manœuvres de l'abbé de Saint-Cyprien de Poitiers pour se faire attribuer l'église Sainte-Croix d'Angles-sur-Anglin, Yves de Chartres cite un passage d'Augustin et quatre extraits de Jérôme, qui affirment la supériorité de l'ordre clérical et l'incapacité des moines à s'occuper des affaires séculières ${ }^{60}$. Le déséquilibre en faveur de Jérôme est justifié par l'auteur lui-même, qui ajoute après la citation d'Augustin: "Mais, comme c'est un clerc qui parle ainsi des clercs, écoutons un moine parler des moines ${ }^{61} »$, confirmant, s'il en était encore besoin, la plus grande autorité de Jérôme en ces matières. L'utilisation de la sentence est plus limitée dans les deux autres textes : la Lettre 41, adressée à l'abbé Geoffroy de Vendôme (10931132), cite la maxime pour expliquer que la bénédiction d'un moine n'est pas un sacrement, tandis que la Lettre 108, adressée à Pascal II (1099-1118), l'évoque en reprochant à l'abbé de Marmoutier d'avoir usurpé le sacerdoce. Mais, dans un cas comme dans l'autre, la sentence hiéronymienne est censée démontrer par elle-même qu'un moine se distingue fondamentalement d'un clerc en ce qu'il ne peut exercer la fonction sacerdotale. Il convient de rappeler qu'avant de monter sur le siège épiscopal de Chartres, Yves avait dirigé la communauté de chanoines réguliers de Saint-Quentin de Beauvais, pour laquelle il rédigea une règle qui servit de modèle à

indications de la p. 115, n. 2, et de l'introduction à l'édition des lettres (éd. cit., n. 55, p. XXVI, n. 1).

59 J. Leclercq, «La collection des lettres d'Yves de Chartres», art. cit., n. 58, p. 113. La première mention d'un volumen epistolarum date cependant d'une période très tardive, puisqu'il s'agit de la chronique d'Aubri de Trois-Fontaines (†1241), qui l'évoque à l'année 1116 (éd. P. Scheffer-Boichorst, Hannover [MGH, SS, 23], 1874, p. 821, 1. 7).

${ }^{60}$ Yves de Chartres, Correspondance, op. cit., n. 55, p. 150 : Augustinus, Ep. LX, 1 etiam bonus-clericum faciat (éd. cit., n. 2, p. 221, 1.22-p. 222, 1. 1); Hieronymus, Adv. Vigil. (fragment 1) ; Id., Ep. XIV, 8 clerici-pascor (éd. cit., n. 3, p. 55, 1. 8-9); Id., Ep. LVIII, 5 si cupis-Macharios (ibid., p. 533, 1.18-p. 534, 1.7); Id., Ep. CXXV, 8 mihi oppidumcensemur (éd. I. Hilberg, Wien-Leipzig [CSEL, 56], 1918, p. 127, 1. 1-2).

${ }^{61}$ Yves de Chartres, Correspondance, op. cit., n. 55, p. 150 : Sed quia clericus ista dixit de clericis, audiamus monachum dicentem de monachis (trad. J. Leclercq, p. 151). 
d'autres établissements ${ }^{62}$. L'adoption par lui de cette sentence de Jérôme s'inscrivait donc aussi dans un contexte clairement réformateur.

Selon Paul Fournier, Yves de Chartres aurait eu accès aux collections canoniques italiennes à l'occasion de l'un de ses deux voyages en Italie ${ }^{63}$. Il s'était en effet rendu une première fois auprès du pape pour se faire consacrer évêque : la cérémonie avait eu lieu en novembre 1090 à Capoue, Urbain II (1088-1099) ayant dû quitter Rome devant l'avancée des troupes impériales ${ }^{64}$. Le nouvel évêque rentre à Chartres au début de l'année 1091, mais retourne à Rome, de novembre 1093 à janvier 1094, après sa captivité dans le donjon d'Hugues du Puiset ${ }^{65}$. Le fragment hiéronymien cité par les collections ivoniennes n'a toutefois pas pu être simplement recopié dans une compilation italienne: Yves extrait un passage bien plus long que ceux contenus dans la Collection en 183 titres (fragment 3) ou dans la version A Aucta d'Anselme de Lucques (fragment 6); il l'identifie surtout à l'Epistola ad Riparium et Desiderium presbiteros, alors que les collections contemporaines privilégiaient la référence à Vigilance ou se contentaient de renvoyer à Jérôme ${ }^{66}$. L'examen précis du contexte de la sentence dans les écrits d'Yves de Chartres semble néanmoins suggérer que son attention aurait pu être attirée par la lecture de la Collection en 183 titres ou d'un proche dérivé, mais qu'il serait ensuite revenu au texte original, afin d'intégrer une version plus personnelle de ce passage à son propre recueil. Ainsi, le canon qui précède immédiatement la sentence de Jérôme dans la Collection en 183 titres est composé de plusieurs extraits d'un autre texte hiéronymien: la Lettre 58 adressée à Paulinus ${ }^{67}$. Or, le dernier fragment de cette

${ }^{62}$ R. Sprandel, Ivo von Chartres und seine Stellung, op. cit., n. 51, p. 143. Un autre exemple de l'activité réformatrice d'Yves, en tant que prévôt de Saint-Quentin de Beauvais, est donné par la lettre qu'il adresse à l'archevêque de Reims, en faveur d'un confrère qui n'avait pas réussi à réformer les Augustins de Saint-Denis de Reims (Fr. S. Schmitt, "Trois lettres inconnues d'Yves de Chartres », Revue bénédictine, 50, 1938, p. 84-88).

${ }^{63}$ P. Fournier, "Yves de Chartres et le droit canonique », Revue des questions historiques, 63, 1898, p. 397, rééd. dans Id., Mélanges de droit canonique, éd. Th. Kölzer, t. I, Aalen, 1983, p. 740.

${ }^{64}$ Cet événement est relaté dans la lettre qu'Urbain II adresse au clergé et au peuple de Chartres, le 24 nov. 1090 (JL 5438).

${ }^{65}$ R. Sprandel, Ivo von Chartres und seine Stellung, op. cit., n. 51, p. 178.

${ }^{66}$ On trouve «in epistola adversus Vigilantium » dans la Collection en 183 titres et dans celle en 5 livres (cf. supra, n. 16 et 19), «in libello adversus Vigilantium hereticum scripto » dans la Defensio Heinrici IV regis (éd. cit., n. 27, p. 442, 1. 2-3), «Hieronimus in epistola » dans l'annexe de la Sinemuriensis (cf. supra, n. 21) et même seulement «Jeronimus» dans la version A Aucta et dans la Collection en 13 livres (cf. supra, n. 25 et 26). Riparius et Desiderius sont les deux prêtres aquitains à qui Jérôme envoie son traité contre Vigilance (CCSL, 79C, p. VI).

${ }^{67}$ Collectio CLXXXIII titulorum, 143, 4: De vita monachorum. JERONIMUS AD PAULINUM EPISCOPUM. Re vera ut simpliciter motus mentis mee fatear, considerans et propositum tuum [...] semper condemnat quod semel contempserit. Et post pauca. Nunc vero summe est stultitie renuntiare seculo, dimittere patriam [...] De toto huc orbe concurrit. Et post pauca. Sin autem cupis esse quod diceris monachus, id est solus, quid facis in urbibus ? [...] vir stans coram Domino (éd. cit., n. 16, p. 227-228). 
lettre dans la compilation italienne correspond exactement à l'incipit du canon qui précède la sentence de Jérôme dans le Décret ${ }^{68}$. Là encore, l'extrait a été remanié par Yves de Chartres, mais la succession, dans ces deux collections, de deux mêmes sources matérielles aux amorces identiques laisse supposer que la lecture de l'une aurait pu orienter les choix de l'autre ${ }^{69}$. Étant donné que la maxime n'apparaît dans la correspondance ivonienne qu'en 1094, date à laquelle elle est citée deux fois, on serait tenté de penser que c'est à l'occasion de son second voyage en Italie qu'Yves de Chartres aurait pris connaissance de ce passage de l'Adversus Vigilantium. Mais il pourrait tout aussi bien s'agir de l'exploitation concrète d'un des dossiers canoniques qui venaient d'être réunis pour composer le Décret $^{70}$.

\section{La diffusion de la sentence dans la première moitié du XII siècle}

\section{La transmission dans les collections canoniques}

Le début du XII ${ }^{\mathrm{e}}$ siècle voit une réaffirmation de la législation conciliaire contre le sacerdoce des moines ${ }^{71}$. En fait, les conciles de cette période se contentent souvent de mettre en application des dispositions déjà prises par Alexandre II (10611073) et Grégoire VII (1073-1085). Ainsi, le concile de Clermont (1095) confie à l'évêque la collation des cures gérées par les monastères ${ }^{72}$. Le concile de Poitiers (1100) interdit même aux moines d'exercer tout ministère paroissial ${ }^{73}$. Quant au concile de Gran (1114), l'actuelle Esztergom en Hongrie, il étend cette interdiction aux abbés ${ }^{74}$. Tout cela aboutit au canon 16 de Latran I (1123), qui retire aux moines le droit de célébrer des messes publiques et d'administrer la pénitence et l'extrême onction $^{75}$. Il n'est donc pas étonnant, dans un tel contexte, que la sentence de Jérôme ait continué à circuler dans les collections canoniques jusqu'au milieu du siècle, date à laquelle elle est finalement intégrée au Décret de Gratien. On peut distinguer trois

\footnotetext{
${ }^{68}$ Ivo Carnotensis, Decretum, 7, 2 : Hieronimus in EPISTOLA AD PAULINUM PRESBITERUM DE INSTITUTIONE CLERICORUM. De monachorum singularitate. Si cupis esse quod diceris, monachus, id est solus [...] nihil cuiquam tribuas, nec filiorum panem canes comedant (éd. cit., n. 48).

${ }^{69}$ Précisons qu'à l'instar de l'Adv. Vigil., ces extraits de la Lettre 58 ne sont cités par aucune compilation canonique, avant la Collection en 183 titres.

${ }^{70}$ On constate en effet que trois des quatre citations hiéronymiennes de la Lettre 36 (cf. supra, n. 60) figurent dans le Livre VII du Décret, dans un ordre presque identique : Adv. Vigil. (= Decretum, 7, 3), Ep. LVIII (= Decretum, 7, 2) et Ep. CXXV (=Decretum, 7, 4). Mais trois des quatre citations se retrouvent aussi au Titre 143 de la Collection en 183 titres : Adv. Vigil. (= 183T, 143, 5), Ep. XIV (=183T, 143, 6), Ep. LVIII (=183T, 143, 4c).

${ }^{71}$ U. Berlière, «L'exercice du ministère », art. cit., n. 1, p. 342.

${ }^{72}$ Conc. Clermont (1095), c. 43 (R. Somerville, The Councils of Urban II, t. I, Amsterdam, 1972, p. 148).

${ }^{73}$ Conc. Poitiers (1100), c. 11 (G. D. Mansi, Sacrorum conciliorum nova et amplissima collectio, t. XX, Venetiis, 1775, col. 1124).

${ }^{74}$ Conc. Gran (1114), c. 38 (ibid., t. XXI, col. 106-108).

${ }^{75}$ Les conciles æecuméniques, dir. G. Alberigo, t. II/1, Paris, 1994, p. 422, 1. 13-16.
} 
traditions textuelles parmi les compilations qui la citent: une première branche remonte plus ou moins directement à la Collection en 183 titres, une deuxième part de la collection canonique dite Polycarpus et une troisième des recueils chartrains, à savoir la Panormie et la Collectio Tripartita.

L'influence de la Collection en 183 titres est aisément repérable, grâce à l'inscription qu'elle attribue au canon: Jeronimus in epistola adversus Vigilantium. C'est elle qu'on retrouve dans la Collection en 7 livres du ms. Wien, ÖNB 2186, qui restitue le même fragment avec deux canons voisins ${ }^{76}$. Composé par un fervent partisan de la réforme, ce recueil fut terminé entre 1112 et 1120, en Italie centrale, peut-être à Rome ou dans le Latium ${ }^{77}$. On peut supposer une filiation identique pour l'annexe de la version $\mathrm{Bb}$ de la collection d'Anselme de Lucques, qui contient le fragment 3 de la sentence, sous la même inscription ${ }^{78}$. Si ce nouveau remaniement de la compilation anselmienne date de la fin $\mathrm{du} \mathrm{XI}^{\mathrm{e}}$ siècle, il n'est en fait conservé que dans un manuscrit copié à Lucques au tout début du XII ${ }^{\mathrm{e}}$ siècle $^{79}$. Surtout, cette version comporte, à partir du fol. $204^{\mathrm{r}}$, une série d'additions dont certaines sont datables. La maxime se lit ainsi au fol. $210^{\mathrm{v}}$, dans une partie du volume recopiée entre 1109 et $1143^{80}$. Elle apparaît, par ailleurs, dans un contexte proche de celui de la Collection en 7 livres $^{81}$. Il faudrait sans doute aussi rattacher à cette origine toscane le fragment 3 cité dans la Collection en 3 livres, même s'il ne présente pas

\footnotetext{
${ }^{76}$ Collectio VII librorum, 5, 52, 1 : IdEM [JERONIMUS] IN EPISTOLA ADVERSUS VigILANTIUM. Monachus non doctoris habet, sed plangentis [...] pavidus prestoletur adventum (Wien, Österreichische Nationalbibl., Lat. 2186, fol. 208 ${ }^{\mathrm{r}}$, d'après L. Fowler-Magerl). Les deux canons qui suivent la sentence de Jérôme dans la Collection en 183 titres sont par ailleurs repris dans les canons qui encadrent le même extrait dans la Collection en 7 livres (183T, 143, $6 \approx 7 \mathrm{~L}, 5,51,1 ; 183 \mathrm{~T}, 143,7=7 \mathrm{~L}, 5,52,2$ ). L'explicit du canon 7L, 5, 51, 1 (die Domini $J e s u$ ) n'appartenant pas à la source matérielle (Hieronymus, Ep. XIV), il n'a pas été possible de déterminer avec exactitude la taille de cet extrait par rapport au canon 183T, 143, 6 .

${ }^{77}$ L. Kéry, Canonical Collections, op. cit., n. 17, p. 269. Il ne subsiste de cette collection que 4 mss. du XII ${ }^{\mathrm{e}}$ s. : Vat. lat. 1346 et Wolfenbüttel, Helmst. 308, pour la première recension ; Wien, ÖNB 2186, et Cortona, BC 43, pour la seconde recension (L. Fowler-Magerl, Clavis Canonum, op. cit., n. 17, p. 233).

${ }^{78}$ Anselmus Lucensis, Collectio canonum (version Bb), App. 33 : JERONIMUS IN EPISTOLA ADVERSUS Vigilantium. Monachus non doctoris habet, sed plangentis [...] pavidus prestoletur adventum (Vaticano, Bibl. Apost. Vat., Barb. lat. 535, fol. 210 , d'après L. Fowler-Magerl).

79 L. Fowler-Magerl, Clavis Canonum, op. cit., n. 17, p. 157. L'unique témoin de ce remaniement (Vat. Barb. lat. 535) a même peut-être été copié par les chanoines réguliers de San Frediano (P. Landau, «Erweiterte Fassungen », art. cit., n. 26, p. 331, rééd. p. 89*).

${ }^{80}$ C'est-à-dire entre une lettre de Pascal II, datée de 1109 (JL 6492), copiée au fol. $206^{\mathrm{r}}$, et une lettre non datée d'Innocent II (1130-1143), copiée par une autre main au fol. 212 ${ }^{\mathrm{v}}$ (L. FowlerMagerl, Clavis Canonum, op. cit., n. 17, p. 158).

${ }^{81}$ Le canon suivant la sentence hiéronymienne dans l'annexe de la version Bb (App. 34) est exactement le même que le canon équivalent dans la Collection en 7 livres $(5,52,2)$.
} 
d'inscription significative ${ }^{82}$. En effet, selon Giuseppe Motta, ce recueil fut compilé à Pistoia, entre 1111 et 1124 , par un partisan de la réforme qui utilisa notamment la Collection en 183 titres $^{83}$. Le fragment contenu dans la Collection en 9 livres du ms. Vat. Arch. S. Pietro, C. 118, appartient à la même tradition, puisque son compilateur s'est contenté de réorganiser la Collection en 3 livres, vers 1123-1125 $5^{84}$. Enfin, on rencontre encore la sentence de Jérôme dans la version C de la collection d'Anselme de Lucques ${ }^{85}$. Mais ce dernier remaniement de la compilation anselmienne - en fait, une variante de la version B-n'est plus conservé que dans trois manuscrits du XVI ${ }^{\mathrm{e}}$ siècle ${ }^{86}$. On ne peut donc pas écarter l'éventualité que cette addition provienne en fait d'une interpolation bien postérieure au XII ${ }^{\mathrm{e}}$ siècle.

Il est difficile d'identifier la source formelle à partir de laquelle Grégoire, cardinal du titre de Saint-Chrysogone $(† 1113)$, a rédigé le canon du Polycarpus qui contient la maxime. Celle-ci apparaît en effet, dès la première version du recueil, sous la forme du fragment 4 , qui n'avait jamais été isolé jusqu'alors ${ }^{87}$. Composée à

${ }^{82}$ Collectio III librorum, 2, 29, 12 : JERONIMUS. Monachus non doctoris habet, sed plangentis officium, quod se vel mundum lugeat et Domini pavidus prestoletur adventum (Collectio canonum trium librorum, t. I, éd. G. Motta, Città del Vaticano, 2005, p. 390).

${ }^{83}$ Ibid., p. XXXV-XXXVI, XXXIX. La collection n'a pas pu être composée avant 1111, puisqu'elle contient un extrait du Liber de honore Ecclesiae que Placido di Nonantola a écrit dans les premiers mois de cette année. On ne conserve cette collection que dans 2 mss. du $\mathrm{XII}^{\mathrm{e}}$ s. : Pistoia, Arch. Cap. del Duomo, C.135 (109); Vat. lat. 3831 (L. Kéry, Canonical Collections, op. cit., n. 17, p. 269-270).

${ }^{84}$ Collectio IX librorum, 5, 5, 12: Jeronimus. Monachus non doctoris, sed plangentis officium [...] pavidus prestoletur adventum (Vaticano, Bibl. Apost. Vat., Arch. di San Pietro, C.118, fol. $64^{\mathrm{vb}}$, d'après L. Fowler-Magerl). Sur la datation de la collection, voir P. Fournier, G. Le Bras, Histoire des collections, op. cit., n. 17, t. II, p. 208. Ce remaniement, peut-être réalisé dans le nord de l'Italie, n'a survécu que dans 2 mss., le Berlin lat. fol. 522 contenant une version plus ancienne que le Vaticano, Arch. S. Pietro C.118 (L. Fowler-Magerl, Clavis Canonum, op. cit., n. 17, p. 235-236).

${ }^{85}$ Anselmus Lucensis, Collectio canonum (version C), 5, 64 : IDEM [Pelagius]. Monachus non doctoris, set plangentis habet $[\ldots]$ prestoletur adventum (P. Landau, «Die Rezension C der Sammlung des Anselm von Lucca », Bulletin of Medieval Canon Law, 16, 1986, p. 33, rééd. dans Id., Kanones und Dekretalen, op. cit. n. 26, p. 59*). À une date indéterminée, un copiste a inséré l'extrait d'une pseudo-décrétale de Pélage II $(\mathrm{JK} \uparrow 1050)$ entre le fragment de l'Adv. Vigil. $(5,64)$ et un extrait de la Lettre 58 de saint Jérôme $(5,62)$, sans prendre la peine de corriger l'inscription de la sentence.

${ }^{86}$ P. Landau, «Die Rezension C», art. cit., n. 85, p. 27-28, rééd. p. 53*-54*.

${ }^{87}$ Polycarpus, 4, 35, 13 : IDEM [Jeronimus]. Monachus non doctoris habet, sed plangentis offitium, qui vel se vel mundum lugeat et Domini pavidus prestoletur adventum, qui sciens inbecillitatem suam et vas fragile quod portat, timeat offendere, ne inpingat et corruat atque frangatur (éd. électronique de H. Fuhrmann et U. Horst, fondée sur le travail préparatoire de C. Erdmann : www.mgh.de/datenbanken/). On compte $12 \mathrm{mss}$. de la première version et un ms. de la seconde version, qui fut sans doute composée peu après 1120 dans le milieu curial romain (L. Kéry, Canonical Collections, op. cit., n. 17, p. 267). La sentence hiéronymienne se trouve aussi dans cette seconde version: Polycarpus, 4, 37, 8 (Paris, Bibl. nat., Lat. 3882, fol. $74^{\mathrm{vb}}$, d'après L. Fowler-Magerl). 
Rome entre 1104 et 1113, cette collection canonique s'inspira des œuvres dont on pouvait disposer à la cour pontificale au début du XII ${ }^{\mathrm{e}}$ siècle $^{88}$. Le cardinal Grégoire utilisa, entre autres, la Collectio Dionysio-Hadriana, les faux pseudo-isidoriens, le Décret de Burchard de Worms, la Collection en 74 titres et la Collection en 183 titres, dans laquelle la sentence est citée pour la première fois ${ }^{89}$. Mais il utilisa aussi la collection d'Anselme de Lucques dans une version qu'il est encore difficile d'identifier ${ }^{90}$. On peut en fait très bien imaginer qu'il ait procédé comme Yves de Chartres, en revenant à la source hiéronymienne pour sélectionner une citation mieux appropriée que celle qu'il avait lue dans une compilation antérieure. Il est probable, en revanche, que le Polycarpus ait inspiré plus ou moins directement la citation de la maxime dans la Collectio Caesarangustana, qui est la seule autre collection canonique à transmettre le fragment sous cette forme ${ }^{91}$. On sait en effet que la première version de ce recueil, composée vers 1120 en Catalogne ou dans le sud de la France, entretient un lien avec le Polycarpus, même si la nature de cette relation reste discutée ${ }^{22}$.

À l'instar de la Collection en 183 titres, l'influence chartraine dans la diffusion de la sentence se reconnaît à l'inscription caractéristique qui précède le canon : Hieronimus in epistola ad Riparium et Desiderium presbiteros. Il en va ainsi de la Collection en 10 parties, qui reprend le même extrait qu'Yves de Chartres avec la même inscription ${ }^{93}$. Cette compilation, rédigée vers 1123 dans le nord de la France, s'inspirait en effet largement de la Panormie ${ }^{94}$. On soupçonne la même

\footnotetext{
${ }^{88}$ Suivant les auteurs, cette collection aurait été achevée entre 1104 et 1106 (P. Fournier, G. Le Bras, Histoire des collections, op. cit., n. 17, t. II, p. 170-171) ou entre 1111 et 1113 (U. Horst, Die Kanonessammlung Polycarpus des Gregor von S. Grisogono. Quellen und Tendenzen, München, 1980, p. 3-6).

${ }^{89}$ U. Horst, Die Kanonessammlung, op. cit., n. 88, p. 9-10 ; complété par G. Motta, « Nuove identificazioni nella collezione canonica detta "Polycarpus" ", Aevum, 57, 1983, p. 232-244.

${ }^{90}$ Le compilateur de la version Bb aurait utilisé les mêmes sources que le cardinal Grégoire, selon U. Horst, Die Kanonessammlung, op. cit. n. 88, p. 45-46.

${ }^{91}$ Collectio Caesaraugustana, 9, 7 : IHERONIMUS. De officio monachi. Monachus non doctoris habet, sed plangentis [...] corruat atque frangatur (Salamanca, Bibl. Univ., 2664, fol. $76^{\text {rb }}$, d'après L. Fowler-Magerl).

${ }^{92}$ Sur l'origine et la date de cette première version, voir L. Fowler-Magerl, Clavis Canonum, op. cit., n. 17, p. 239-242. Sur ses liens avec le Polycarpus, voir M. Brett, "The sources and influence of Paris, Bibliothèque de l'Arsenal MS 713 ", Proceedings of the Ninth International Congress of Medieval Canon Law, Munich, 13-18 July 1992, éd. P. Landau, J. Müller, Città del Vaticano, 1997, p. 166-167. On conserve 5 mss. de la première version, 4 de la deuxième et un seul de la troisième (L. Kéry, Canonical Collections, op. cit., n. 17, p. 260-261).

93 Collectio Xpartium, 3, 52, 3, 4: Idem [Jeronimus] AD Riparium et Desiderium PRESBITEROS. Monachus non doctoris, sed plangentis habet [...] perdam aliquando victoriam (Firenze, Bibl. Naz., Conv. soppr., D.II.1476, fol. 84 ${ }^{\mathrm{r}-\mathrm{v}}$, d'après L. Fowler-Magerl).

${ }^{94}$ Ce remaniement de la Collection en 9 livres aurait été réalisé par l'archidiacre Gauthier de Thérouanne, si l'on suit l'hypothèse du chanoine De Smet (L. Waelkens, D. Van den Auweele, «La collection de Thérouanne en IX livres à l'abbaye de Saint-Pierre-au-MontBlandin: le codex Gandavensis $235 »$, Sacris Erudiri, 24, 1980, p. 121-122). On conserve
} 
origine ivonienne dans la collection dite «Ambrosiana II» du ms. Milano, Ambrosiana I.145 inf., composée à Milan dans la première moitié du XII ${ }^{\mathrm{e}}$ siècle $^{95}$. La maxime n'est cependant pas citée à partir de la Panormie, mais de la Collectio Tripartita, comme l'attestent la variante de l'incipit et le découpage des deux canons suivants ${ }^{96}$. Ce constat confirme, à tout le moins, l'hypothèse de Peter Landau, pour qui la Collectio Ambrosiana II serait le résultat d'une modernisation d'anciennes compilations milanaises à partir de la Tripartita ${ }^{97}$. Si la part de la tradition ivonienne paraît modeste dans la diffusion de la sentence au sein des collections canoniques, il ne faut pas oublier que le nombre de copies de la Tripartita et de la Panormie dépasse de très loin celui des compilations italiennes qui citent le passage ${ }^{98}$. C'est néanmoins le Bolonais Gratien qui assure à ce texte sa véritable postérité. Héritier des traditions italienne et chartraine, il est parmi les derniers canonistes à citer la sentence de Jérôme. Elle figure ainsi au début de la Causa XVI de son Décret, sous la forme d'un fragment sans doute extrait de la Collectio Tripartita, mais réduit au format courant des collections italiennes ${ }^{99}$. Elle apparaît surtout dès la première version du Décret, qui fut rédigée vers 1139 et rend davantage compte de l'enseignement du maître ${ }^{100}$. Si un tel aboutissement a eu tendance à éclipser tous les autres relais dans la transmission ultérieure de la sentence, le rôle d'Yves de Chartres n'en reste pas moins essentiel pour la première moitié du XII siècle, car il permet le transfert de la maxime du monde des canonistes à celui des théologiens.

6 mss. de cette collection, qui fut copiée jusqu'au XIII ${ }^{\mathrm{e}}$ s. (L. Kéry, Canonical Collections, op. cit., n. 17, p. 263).

${ }^{95}$ Collectio Ambrosiana II, 10 : ITEM In EPISTOLA AD RIPARIUM ET DeSiderium PRESBITERos. JERONIMUS. Monachus non doctoris, sed plangentis habeat $[. .$.$] perdam aliquando victoriam$ (Milano, Bibl. Ambrosiana, I.145 inf., fol. 25' , d'après L. Fowler-Magerl). L'analyse de ce recueil a été réalisée par G. Picasso, Collezioni canoniche milanesi del secolo XII, Milano, 1969, p. 81-143. Pour une mise au point récente, voir L. Fowler-Magerl, Clavis Canonum, op. cit., n. 17, p. 124-126.

${ }^{96}$ Le c. 10 de la Collectio Ambrosiana II présente la variante d'incipit de la Tripartita (cf. supra, n. 50). Par ailleurs, les c. 11 et 12 citent deux extraits de la Lettre 125 de Jérôme sous la forme adoptée par le Décret, puis la Tripartita, 3, 11, 3 (Mihi oppidum-ingressus est) et 4 (Numquam de manibus-pedes suos), et non sous celle transmise par la Panormie, 3, 177 (Mihi oppidum - in manu habentes) et 178 (Facito aliquid-pedes suos).

${ }^{97}$ P. Landau, « Kanonessammlungen in der Lombardei im frühen und hohen Mittelalter », Atti dell'XI Congresso internazionale di studi sull'alto Medioevo, Spoleto, 1989, p. 452 et 456, rééd. dans Id., Kanones und Dekretalen, op. cit., n. 26, p. 464* et 468*.

${ }^{98}$ On conserve près de 170 mss. de la Tripartita et de la Panormie, alors qu'on ne compte qu'une quarantaine de témoins pour la tradition italienne, toutes origines confondues.

${ }_{99}$ Gratianus, Decretum, C. 16, q. 1, c. 4: Monachus non habet docentis offitium sed plangentis. ITEM JERONIMUS AD RIPARIUM ET Desiderium. Monachus non doctoris, sed plangentis habet offitium, qui vel se vel mundum lugeat et Domini pavidus prestoletur adventum (éd. E. Friedberg, t. I, Leipzig, 1879, col. 762). Si la taille du fragment renvoie à la tradition textuelle de la Collection en 183 titres, l'inscription est, quant à elle, typiquement chartraine, et l'extrait contient surtout une variante propre à la Tripartita (cf. supra, n. 50).

${ }^{100}$ A. Winroth, The Making of Gratian's Decretum, Cambridge, 2000, p. 215. 


\section{L'usage de la sentence par les théologiens}

On aurait tort de cantonner Yves de Chartres au rôle réducteur de «premier canoniste». Élève de Lanfranc du Bec, au côté d'Anselme de Cantorbéry, il possédait une solide culture théologique et patristique ${ }^{101}$. À une époque où la frontière entre le droit canonique et la théologie n'était pas définitivement fixée, l'évêque de Chartres était avant tout perçu par ses contemporains comme un savant capable de fournir des solutions doctrinales à des problèmes concrets, comme il le fit, du reste, dans le domaine matrimonial, qui touchait tout autant au droit qu'à la théologie morale. Cette capacité d'expertise explique que ses œuvres ont non seulement exercé une forte influence sur les canonistes, tel Alger de Liège, mais ont aussi pu servir de sources pour des théologiens comme Pierre Abélard ou Hugues de Saint-Victor ${ }^{102}$. Ses lettres et ses sermons étaient d'ailleurs transmis avec des florilèges patristiques et des écrits parfois reliés à l'école de Laon ${ }^{103}$. La notoriété d'Yves en matière théologique était d'autant plus reconnue qu'un certain nombre de sentences lui avaient été faussement attribuées, comme le prouve l'analyse critique du Liber pancrisis, compilation de textes d'inspiration laonnoise rédigée après $1140^{104}$. Dans ce recueil, l'autorité d'Yves était artificiellement élevée au rang de celles de Guillaume de Champeaux, d'Anselme et de Raoul de Laon, dans une sorte de parallèle avec les quatre Pères latins : Augustin, Jérôme, Ambroise et Grégoire ${ }^{105}$. C'est dire toute l'importance que la théologie du premier XII ${ }^{\mathrm{e}}$ siècle accordait à son

${ }^{101}$ R. Sprandel, Ivo von Chartres und seine Stellung, op. cit., n. 51, p. 12.

${ }^{102}$ Alger de Liège utilisa la Panormie comme une source doctrinale, canonique et patristique (R. Kretzschmar, Alger von Lüttichs Traktat "De misericordia et iustitia». Ein kanonistischer Konkordanzversuch aus der Zeit des Investiturstreits, Sigmaringen, 1985, p. 27-28 et 105-114). Dans le De sacramentis christianae fidei d'Hugues de Saint-Victor, la plupart des citations canoniques à propos du mariage proviennent plus ou moins directement des œuvres d'Yves de Chartres (P. Fournier, «Les collections canoniques attribuées à Yves de Chartres », BEC, 58, 1897, p. 658, rééd. dans Id., Mélanges de droit canonique, op. cit., n. 63, t. I, p. 660). Quant à Pierre Abélard, il connaissait aussi les collections chartraines et leur a emprunté un certain nombre de textes pour composer son Sic et Non, même s'il les a complétées en revenant aux sources originales (ibid., p. 663-664, rééd. p. 665-666).

${ }^{103}$ Il s'agit des ms. Paris lat. 3004 (où les lettres sont précédées d'un florilège patristique et de questions théologiques), Paris lat. 2887A (où elles sont suivies d'un traité sur le mariage) et Paris lat. 2485 (où elles accompagnent des textes liés à l'école de Laon), selon J. Leclercq, «La collection des lettres d'Yves de Chartres », art. cit., n. 58, p. 116. Précisons toutefois que seul le ms. Paris lat. 3004 figure parmi les florilèges anselmiens - et encore à faible présence anselmienne-, selon la typologie de C. Giraud, "Per verba magistri ». Anselme de Laon $(† 1117)$ et son école, Turnhout, sous presse, chap. 3 , § 3c.

${ }^{104}$ Sur les 28 sentences attribuées à Yves de Chartres par les rubriques du Liber pancrisis, seules deux se révèlent provenir effectivement de lui (C. Giraud, "Per verba magistri », op. cit., n. 103, chap. 3, § 2a). Pour l'origine et la datation de ce recueil, voir ibid., chap. 3, $\S 2$ b. Les prétendues sentences ivoniennes ont été éditées par Fr. Pl. Bliemetzrieder, «Zu den Schriften Ivos von Chartres (†1116). Ein literargeschichtlicher Beitrag», Sitzungsberichte der kaiserlichen Akademie der Wissenschaften in Wien. Philos.-hist. Klasse, 182/6, 1917, p. 55-71.

${ }^{105}$ C. Giraud, «Per verba magistri », op. cit., n. 103, chap. 3, § 2a. 
œuvre. Il n'est donc pas étonnant de retrouver la maxime, qu'Yves de Chartres avait tant contribué à populariser, dans un florilège lié à l'école de Laon, comme la collection du ms. Kremsmünster, Stiftsbibl., CC 280, recopiée au XIII ${ }^{\mathrm{e}}$ siècle en Bavière ou en Autriche ${ }^{106}$. Mais 1'utilisation de la sentence par les théologiens remonte en fait au début des années 1120 .

Le théologien qui fit le plus large usage de cette maxime est Rupert de Deutz $(\dagger 1129)$, qui la cite à six reprises dans ses écrits, à chaque fois pour la récuser. Ce moine bénédictin avait dû quitter l'abbaye Saint-Laurent de Liège en 1116, après la controverse qui l'avait opposé à Anselme de Laon au sujet de la doctrine de la prédestination ${ }^{107}$. Réfugié à l'abbaye de Siegburg, où il bénéficiait de la protection de l'archevêque de Cologne et de l'abbé Kuno, Rupert se lança en 1119 dans un autre combat : le droit des moines à prêcher et enseigner publiquement dans l'Église. Mais il n'est pas impossible que ce nouvel engagement soit relié au précédent, dans la mesure où les disciples liégeois d'Anselme de Laon s'étaient moqués de la prétention de ce moine à discuter l'enseignement d'un maître qu'il n'avait jamais entendu. À l'occasion de la polémique, la sentence de Jérôme, connue des Laonnois grâce aux œuvres d'Yves de Chartres, avait certainement dû être opposée à Rupert. Il reste que le premier texte qu'il publia à Cologne se fondait essentiellement sur la réfutation de cette maxime. Avec seize manuscrits conservés, l'Altercatio monachi et clerici quod liceat monacho predicare est l'un des traités rupertiens les plus connus $^{108}$. Rédigé entre 1119 et 1122 , sous la forme d'une joute verbale, il en a gardé le ton abrupt et un latin proche du langage parlé. L'Altercatio se fondait sans doute sur un débat qui avait réellement eu lieu, mais l'identité du clerc-l'adversaire de Rupert - et les circonstances de leur dispute ne sont pas clairement définies ${ }^{109}$. La

\footnotetext{
${ }^{106}$ Il s'agit d'un ms. composite au contenu principalement théologique, la collection de sentences occupant la sixième partie du vol. (fol. $198^{\mathrm{r}}-217^{\mathrm{v}}, 3^{\mathrm{e}}$ tiers $\mathrm{XIII}^{\mathrm{e}} \mathrm{s}$.). La maxime de Jérôme (fol. $214^{\text {ra }}$ ) est insérée entre un extrait du sermon De sacramentis dedicationis d'Yves de Chartres (PL 162, col. 527B-D) et des sentences anselmiennes, tirées notamment du Liber pancrisis (H. Fill, Katalog der Handschriften des Benediktinerstiftes Kremsmünster, t. I, Wien, 1984, p. 318).

${ }^{107}$ Dans son De voluntate Dei (1116), Rupert de Deutz s'inquiétait des erreurs théologiques transmises par des élèves d'Anselme de Laon et de Guillaume de Champeaux. Entre la fin de l'année 1116 et le printemps 1117, Anselme intervient directement dans la polémique en envoyant sa fameuse lettre à Héribrand, abbé de Saint-Laurent de Liège (C. Giraud, "Per verba magistri », op. cit., n. 103, chap. 2, § 2c).

108 PL 170, col. 537-542. Aux 15 mss. répertoriés par R. Haacke («Nachlese zur Überlieferung der Schriften Ruperts von Deutz», Deutsches Archiv, 26, 1970, p. 539-540), il faut ajouter le ms. indiqué par M. Bernards (compte rendu dans Zeitschrift für Kirchengeschichte, 84, 1973, p. 111).

${ }^{109}$ Il s'agirait peut-être de Norbert de Xanten, fondateur de l'ordre de Prémontré, qui visita justement Cologne en 1121 et 1122 . Le fait est qu'une copie du XII ${ }^{\mathrm{e}} \mathrm{s}$. intitule le traité Conflictus Ruodperti Coloniensis abbatis [...] cum Noperto clerico, si liceat monacho praedicare an non (München Clm 27129). Il faut toutefois préciser que le ms. London, BL, Add. 10957, porte le titre Disputatio Rodberti abbatis contra Willehelmum diaconum, et que la copie moderne du ms. Wolfenbüttel, Extravagantes 264.37, s'intitule Altercatio Ruperti
} 
sentence hiéronymienne y est, quoi qu'il en soit, produite quatre fois, sous la forme du fragment 1 , parmi d'autres citations antimonastiques ${ }^{110}$. Rupert déjoue l'objection en rappelant que saint Jérôme, lui-même, fut moine et docteur de l'Eglise, qu'il n'y a pas d'incompatibilité entre la cléricature et la profession monastique, et que le droit de prêcher des clercs provient, non de leur état, mais de l'ordination sacerdotale qu'ils reçoivent de l'évêque. La maxime est, une nouvelle fois, réfutée dans la lettre que Rupert, devenu abbé de Deutz, écrit vers 1123-1124 à l'abbé Eberhard de Brauweiler (1110-1126) ${ }^{111}$. On la retrouve enfin au centre d'une quaestio attribuée à un certain «Růdbertus» dans le ms. München Clm 22225, provenant de l'abbaye de Windberg dans l'est de la Bavière ${ }^{112}$.

Cette Questio utrum monachis liceat predicare, que l'on a longtemps crue de Rupert de Deutz, s'avère être en fait du moine Honorius Augustodunensis († vers 1157), reclus près de Ratisbonne ${ }^{113}$. Ce texte est d'ailleurs accompagné, dans le manuscrit de Windberg, d'un traité d'Honorius, intitulé Quod monachis liceat predicare, qui développe les mêmes thèses en partant, lui aussi, du précepte de Jérôme ${ }^{114}$. La diffusion de la sentence en Bavière est probablement due à l'action de l'abbé Kuno, qui emporta sans doute avec lui des écrits de Rupert, lorsqu'il devint évêque de Ratisbonne (1126-1132) ${ }^{115}$. La Questio utrum monachis liceat predicare date certainement des mêmes années que le traité Quod monachis liceat predicare, qui fut écrit après 1126 , et plus vraisemblablement vers $1130-1132^{116}$. Le dernier avatar de cette tradition bavaroise se retrouve dans l'œuvre d'Idung de Prüfening, qui cite au moins cinq fois la sentence. Le parcours de cet auteur illustre un autre débat qui faisait rage au même moment, celui opposant l'ancien et le nouveau

monachi contra Timundum clericum (J. H. Van Engen, Rupert of Deutz, Berkeley, 1983, p. 311-312).

${ }^{110}$ PL 170, col. 538C, 538D, 540C et 540D.

${ }^{111} \mathrm{Ce}$ dernier voulait savoir s'il pouvait confier des églises paroissiales à des moines. La sentence y est citée deux fois, sous la forme du fragment 1 (G. Eckertz, "Chronicon Brunwylrense », Annalen des historischen Vereins für den Niederrhein, 17, 1866, p. 138). Sur l'abbatiat d'Eberhard, voir E. Wisplinghoff, Die Benediktinerabtei Brauweiler, Berlin (Germania Sacra, nouv. sér., 29/5), 1992, p. 40-41 et 188. Sur la datation de la lettre, voir J. H. Van Engen, Rupert of Deutz, op. cit., n. 109, p. 312.

${ }^{112} \mathrm{La}$ sentence y est citée deux fois sous la forme du fragment 1 (J. A. Endres, Honorius Augustodunensis. Beitrag zur Geschichte des geistigen Lebens im 12. Jahrhundert, KemptenMünchen, 1906, p. 145 et 146). Le ms. date du XII ${ }^{\mathrm{e}}$ s.

${ }^{113}$ Cette quaestio se rattache à l'œuvre d'Honorius, tant par la langue et le genre littéraire adoptés que par les nombreuses citations d'origine honorienne (M.-O. Garrigues, «L'œuvre d'Honorius Augustodunensis : inventaire critique », Abhandlungen der braunschweigischen wissenschaftlichen Gesellschaft, 40, 1988, p. 155-156).

${ }^{114}$ Ce traité n'apparaît dans aucune des listes d'œuvres d'Honorius, mais l'étude philologique et la comparaison avec d'autres textes du même auteur ne laissent planer aucun doute sur son origine honorienne (M.-O. Garrigues, «L'œuvre d'Honorius Augustodunensis », art. cit., n. 113, p. 154-155). La sentence y est citée sous la forme du fragment 1 (J. A. Endres, Honorius Augustodunensis, op. cit., n. 112, p. 147).

${ }^{115}$ J. H. Van Engen, Rupert of Deutz, op. cit., n. 109, p. 312.

${ }^{116}$ M.-O. Garrigues, «L'œuvre d'Honorius Augustodunensis », art. cit., n. 113, p. 159, 177. 
monachisme: écolâtre à Ratisbonne, depuis 1133 au moins, Idung rentra au monastère de Prüfening vers 1144, mais, déçu par l'observance clunisienne, il devint cistercien en 1154 ou $1155^{117}$. Lorsqu'il écrit son Argumentum super quatuor questionibus (entre 1132 et 1145), Idung s'inscrit encore dans la lignée de Rupert de Deutz : il rédige un éloge de la profession monastique pour convaincre son collègue Herbord, écolâtre de Bamberg, d'entrer en religion. Mais, lorsqu'il compose son Dialogus duorum monachorum (vers 1155), Idung a déjà changé d'observance : il se lance alors dans une critique en règle du monachisme traditionnel, sous la forme d'un dialogue entre un cistercien et un clunisien. $\mathrm{Si}$, dans les deux cas, la maxime de Jérôme est réfutée au nom de la charge antimonastique qu'elle recèle, le Dialogus montre une plus grande distance de l'auteur vis-à-vis du débat qui opposait les moines aux chanoines réguliers ${ }^{118}$.

Diffusée au nord des Alpes par Yves de Chartres, la sentence n'a pas tardé à être utilisée par les théologiens français. En réalité, elle fit irruption, dès le début du $\mathrm{XII}^{\mathrm{e}}$ siècle, dans le conflit frontal qui opposa Pierre Abélard $(\dagger 1142)$ à l'un de ses anciens maîtres, Roscelin de Compiègne (†vers 1120). Ce dernier avait été condamné à Soissons en 1092 pour sa théorie de la Trinité, ce qui l'avait obligé à quitter le royaume ${ }^{119}$. En 1120, ayant subi les attaques de Roscelin au sujet de sa doctrine trinitaire, Abélard dénonça ce dernier à l'évêque de Paris et demanda qu'on réunît une assemblée ecclésiastique pour trancher leur différend. À l'approche du concile finalement convoqué à Soissons (avril 1121), Roscelin lança une violente diatribe contre son ancien élève, qui avait continué à enseigner, bien qu'il fût devenu moine. Cette lettre se terminait par le rappel, sans fard, de la récente castration d'Abélard. Surtout, elle insistait sur la contradiction entre son état et la poursuite de son enseignement: "Ainsi, une fois l'habit pris, tu as usurpé la fonction de docteur en enseignant des mensonges. Mais tu as cessé, de toute façon, d'être un moine, car saint Jérôme, moine lui-même définissant le moine, dit qu'un moine n'a pas pour fonction d'instruire, mais de pleurer sur lui-même ou le monde et d'attendre en tremblant la venue du Seigneur $\gg{ }^{120}$. Cette citation tranche avec l'usage traditionnel

${ }^{117}$ R. B. C. Huygens, «Le moine Idung et ses deux ouvrages : "Argumentum super quatuor questionibus" et "Dialogus duorum monachorum" ", Studi medievali, $3^{\mathrm{e}}$ sér., 13, 1972, p. 296-298.

${ }^{118}$ La sentence est citée deux fois dans l'Argumentum (R. B. C. Huygens, « Le moine Idung », art. cit., n. 117, p. 364, 1.765-766; p. 366, 1. 834-835) et trois fois dans le Dialogus (ibid., p. $426,1.720-721$; p. $430,1.843-844$ et 849 ), sans compter une occurrence qui n'en reproduit que l'amorce (ibid., p. 428, 1. 783).

${ }^{119}$ Sur la carrière de Roscelin, voir F. Picavet, Roscelin, philosophe et théologien, Paris, 1911. Sur sa doctrine théologique, voir C. J. Mews, «Nominalism and Theology before Abaelard : New Light on Roscelin of Compiègne », Vivarium, 30, 1992, p. 4-33.

${ }^{120}$ Roscelinus, Epistola ad Abaelardum: Quia igitur suscepto habitu doctoris officium mendacia docendo usurpasti, utique monachus esse cessasti, quia beatus Hieronymus monachum, monachus ipse, diffiniens: "Monachus, inquit, non doctoris, sed plangentis habet officium, qui se vel mundum lugeat et Domini pavidus praestoletur adventum» (J. Reiners, «Der Nominalismus in der Frühscholastik. Ein Beitrag zur Geschichte der Universalienfrage im Mittelalter », Beiträge zur Geschichte der Philosophie des Mittelalters, $8 / 5,1910$, p. $80,1.1-5)$. 
qu'en fait Rupert de Deutz au même moment. Jamais la maxime hiéronymienne n'avait aussi clairement stigmatisé la fonction scolaire du moine, en isolant, au sein de la prédication, l'enseignement magistral de la foi. Certes, l'attaque visait Abélard, qui n'était pas un moine ordinaire. Mais force est de constater que, vers 1120, un théologien français pouvait traduire l'officium doctoris de l'Adversus Vigilantium par la fonction de maître et plus seulement par celle de prédicateur, ce qui en dit autant sur l'évolution de la sentence que sur l'émergence d'une autorité magistrale. Roscelin n'était cependant pas le seul à interpréter ainsi le texte de Jérôme.

Dans le Didascalicon, écrit avant 1125, Hugues de Saint-Victor ( $† 1141)$ explique au novice qu'il ne doit pas sauter les étapes de son apprentissage, c'est-àdire philosopher et enseigner, alors qu'il n'est qu'un moine ${ }^{121}$. La maxime se présente ici sous la forme d'une réminiscence - l'autorité de Jérôme n'y est pas alléguée-, ce qui permet à Hugues de décontextualiser la citation et de jouer pleinement sur l'ambivalence du verbe docere. Il en va de même pour Bernard de Clairvaux (†1153), qui proclame son ignorance, dans la Lettre 89 (carême 1125), avant de répondre à son ami Ogier ${ }^{122}$. On reconnaît, là encore, une réminiscence du texte hiéronymien, qui donne l'occasion à saint Bernard d'arborer une modestie toute monastique vis-à-vis de l'activité enseignante ${ }^{123}$. Il faut toutefois préciser qu'énoncé dans ce contexte, le précepte de Jérôme n'a plus, pour ces auteurs, de portée absolue. L'un comme l'autre admettent qu'on y déroge, à condition, pour Hugues, d'avoir fini sa formation ou, pour saint Bernard, d'en avoir reçu la mission. En dehors de Roscelin, l'usage de la sentence par les théologiens français se révèle donc assez peu polémique. Bernard de Clairvaux l'évoque encore à deux reprises dans le Sermon 64 sur le Cantique des Cantiques (entre 1139 et 1143 ) ${ }^{124}$ et dans la

\footnotetext{
${ }^{121}$ Hugo de Sancto Victore, Didascalicon de studio legendi, 5, 8 : Si monachus es, quid facis in turba? Si amas silentium, cur declamantibus assidue interesse delectat? Tu semper jejuniis et fletibus insistere debes, et tu philosophari quaeris? Simplicitas monachi philosophia ejus est. "Sed docere, inquis, alios volo». Non est tuum docere, sed plangere (Ch. H. Buttimer, Hugonis de Sancto Victore Didascalicon de studio legendi. A Critical Text, Washington, 1939, p. 108, 1.24-p. 109, 1. 1). Pour la datation du traité, voir le tableau synoptique de D. Van den Eynde, Essai sur la succession et la date des écrits de Hugues de Saint-Victor, Roma, 1960, pl. h.-t.

${ }^{122}$ Ogier était alors chanoine régulier au Mont-Saint-Éloi, près d'Arras. Il devient, en 1126, abbé de Saint-Médard, par la suite Saint-Nicolas-des-Prés, à Tournai (Bernard de Clairvaux, Lettres, t. II, trad. H. Rochais, Paris, 2001, p. 450-451, n. 1).

${ }^{123}$ Bernardus Claraevallensis, Ep. LXXXIX, 2 : Siquidem vel monachi quod esse videor, vel peccatoris quod sum, officium non est docere, sed lugere. Indoctus quoque, quod et vere me fateor esse, si praesumat docere quod nescit, nihil indoctius agit. Docere itaque nec indocto est in promptu, nec monacho in ausu, nec paenitenti in affectu (Sancti Bernardi Opera, t. VII, éd. J. Leclercq, H. Rochais, Roma, 1974, p. 236, 1. 2-6). Saint Bernard finit, tout de même, par donner à Ogier la réponse qu'il souhaitait.

${ }^{124}$ Bernardus Claraevallensis, Sermones in Cantica Canticorum, 64, 3 : Et scimus monachi officium non docere esse, sed lugere [...] Ex his nempe claret et certum est, quod publice praedicare nec monacho convenit, nec novitio expedit, nec non misso licet (Sancti Bernardi Opera, t. II, éd. J. Leclercq, C.-H. Talbot, H. Rochais, Roma, 1958, p. 168, 1. 3-7). Sur la
} 
Lettre 365 à l'archevêque de Mayence $(1146)^{125}$. Revenant à une lecture plus traditionnelle, le cistercien ne dénonce pas l'enseignement magistral, mais la prédication des moines et, encore, de ceux qui n'y sont pas préparés ou agissent sans mandat. S'il ne fut pas le pourfendeur du monde scolaire qu'on a trop souvent décrit, saint Bernard n'avait pas non plus vocation à défendre l'enseignement monastique ${ }^{126}$. Même Pierre Abélard, qui fut pourtant la première victime de l'emploi polémique de cette maxime en France, ne rechigne pas à la citer comme un précepte moral, non sans l'avoir expurgée de toute charge négative à l'égard de sa propre situation : dans le Sermon 33, qu'il écrit lorsqu'il passe du Paraclet à Saint-Gildas-de-Rhuys (vers 1126-1127), la sentence lui sert à dénoncer les moines qui, comme Norbert de Xanten, accèdent aux fonctions épiscopales ${ }^{127}$, tandis que, dans la solution qu'il donne au $14^{e}$ Problème d'Hélö̈se (vers 1137-1138), seule la seconde partie de la proposition est mise en valeur, afin d'exalter l'affliction monastique ${ }^{128}$. On comprend aisément qu'ayant continué à enseigner durant une bonne partie de la période, il n'ait pas insisté sur l'acception scolaire de l'officium doctoris. C'était le moins que pût faire cet admirateur de l'œuvre de Jérôme ${ }^{129}$.

datation du sermon, voir Bernard de Clairvaux, Sermons sur le Cantique, t. IV, trad. P. Verdeyen, R. Fassetta, Paris, 2003, p. 21.

${ }^{125}$ Bernardus Claraevallensis, Ep. CCCLXV, 1 : Homo ille, de quo agitur in litteris vestris, neque ab homine, neque per hominem, sed neque a Deo missus venit. Quod si se monachum aut eremitam jactat, et ex eo sibi assumit libertatem vel officium praedicationis, potest scire, et debet, quod monachus non habet docentis, sed plangentis officium, quippe cui oppidum carcer esse debet et solitudo paradisus (Sancti Bernardi Opera, t. VIII, éd. J. Leclercq, H. Rochais, Roma, 1977, p. 320, 1. 14 - p. 321, 1. 4). Ce moine Radulfus prêchait le massacre des juifs dans la vallée du Rhin, selon Otton de Freising (Gesta Friderici, I, 38).

${ }^{126}$ Sur les rapports de saint Bernard avec le monde des écoles, voir J. Verger, « Le cloître et les écoles », Bernard de Clairvaux. Histoire, mentalités, spiritualité, Paris, 1992, p. 459-473.

${ }^{127}$ Petrus Abaelardus, Sermo XXXIII : Quo ergo quisque de monastico coetu assumptus circa curam uxoris et domesticorum minus probatus est, magis ad episcopatum accedere trepidet. Qui etiam doctoris officium quanto minus est expertus, juxta illud Hieronymi : "Monachus non doctoris, sed plangentis officium habet, qui se mundum lugeat et Domini pavidus praestoletur adventum », tanto magis ignorat quid populum doceat, qui a populo jam toto tempore sequestratus vixit (PL 178, col. 603A-B). Sur la datation et le contexte du Sermon 33, voir C. Mews, «On Dating the Works of Peter Abelard», Archives d'histoire doctrinale et littéraire du Moyen Âge, 60, 1985, p. 124.

${ }^{128}$ Petrus Abaelardus, Problemata Heloissae, 14 : Beati qui lugent. Luctus salubris proprie convenit monachis, sive ille sit poenitentiae de peccato, sive dilationis a regno [...] Quantum autem de peccatis tam suis quam aliorum lugere conveniat monachum, Hieronymus hujus professionis maximus profitetur dicens: "Monachus non doctoris, sed plangentis habet officium, qui se et mundum lugeat et Domini pavidus praestoletur adventum ». Quid enim vita monastica, nisi quaedam est districtioris poenitentiae forma? (PL 178, col. 699D-700A). Sur la datation des Problemata Heloissae, voir C. Mews, «On Dating the Works of Peter Abelard », art. cit., n. 127, p. 132.

${ }^{129}$ C. J. Mews, «Un lecteur de Jérôme au XII ${ }^{\mathrm{e}}$ siècle : Pierre Abélard », Jérôme entre l'Occident et l'Orient, éd. Y.-M. Duval, Paris, 1988, p. 429-444. 


\section{L'affaiblissement de la sentence}

$\mathrm{Au}$ milieu du XII ${ }^{\mathrm{e}}$ siècle, on constate un net recul de l'usage du précepte hiéronymien. Ce phénomène accompagne en fait l'extinction progressive de la controverse sur l'enseignement ou la prédication des moines. Les attaques des clercs séculiers et des chanoines réguliers avaient en effet provoqué la riposte de plusieurs représentants de l'ordre monastique. Le combat mené par Rupert de Deutz et ses épigones bavarois et liégeois avait, semble-t-il, fini par porter ses fruits ${ }^{130}$. Au cours du XII ${ }^{\mathrm{e}}$ siècle, un modus vivendi s'établit donc entre moines et chanoines réguliers. Gerhoch de Reichersberg ( $\dagger$ 1169), l'ardent propagateur de la vie commune parmi les clercs, reconnaît ainsi, dans son Opusculum de edificio Dei (1128), que les moines ordonnés, étant moines et clercs, peuvent également remplir des fonctions cléricales $^{131}$. De même, Arno de Reichersberg († 1175), qui suit l'engagement de son frère dans la réforme cléricale, souhaite que soit réalisée l'union entre l'ordre canonial et l'ordre monastique dans son Scutum canonicorum (vers 1146-1147) ${ }^{132}$. Enfin, bien qu'il s'oppose directement à l'Altercatio de Rupert de Deutz dans son De institutione clericorum (vers 1150), Philippe de Harvengt (†1183), abbé prémontré de Bonne-Espérance, finit par concéder qu'un moine peut remplacer un clerc et avoir charge d'âme ${ }^{133}$. Cette voie moyenne est assez bien résumée par l'une des versions apocryphes de la lettre d'Anselme de Laon à l'abbé Héribrand de SaintLaurent de Liège, qui admet que les moines puissent exercer une charge pastorale en cas de nécessité ${ }^{134}$. Dans ce nouveau contexte, la sentence de Jérôme ne fait plus polémique. Si elle est encore citée, elle n'est plus qu'une référence convenue à un

${ }^{130}$ Les arguments rupertiens sont en effet repris dans des textes qui ont longtemps été attribués à Rupert de Deutz: la lettre au chanoine Liézelin (PL 170, col. 663-668) s'avère avoir été écrite vers 1130 par l'abbé Wazelin de Saint-Laurent de Liège (Ch. Dereine, « Le problème de la cura animarum », art. cit., n. 1, p. 315, n. 42), tandis que le traité De vita vere apostolica (PL 170, col.611-664) semble devoir être classé parmi les œuvres d'Honorius Augustodunensis (M.-O. Garrigues, «L'œuvre d'Honorius Augustodunensis », art. cit., n. 113, p. 160-161), mais des doutes subsistent sur cette attribution (V. I. J. Flint, Honorius Augustodunensis of Regensburg, Aldershot [Authors of the Middle Ages, 6], 1995, p. 57-58).

${ }_{131}$ Gerhoh Reichersbergensis, Opusculum de edificio Dei, 162 (éd. E. Sackur, Hannover [MGH, L. d. l., 3], 1897, p. 193).

${ }^{132}$ Arno Reichersbergensis, Scutum canonicorum regularium (PL 194, col. 1522D-1523A).

${ }^{133}$ Philippus de Harvengt, De institutione clericorum, IV, De continentia clericorum, § 80, 81, 85 et 86 (PL 203, col. 773B-775B, 779A-783B).

${ }^{134} \mathrm{La}$ formule adoptée rend d'ailleurs un discret hommage à la sentence de Jérôme : Clerici electi sunt ad praedicandum et ad docendos subditos, monachi vero ad orandum, quia clerici propter distractiones officii et negotiorum jugiter orationi vacare non poterant. Tamen causa necessitatis ex praecepto episcopi saepe monachi assumunt officium praedicandi et docendi (PL 162, col. 1590B-C). Ce passage a en fait été rajouté à la lettre originale à une date indéterminée après 1117, comme l'ont déjà noté O. Lottin (Psychologie et morale aux XII et XIII ${ }^{e}$ siècles, t. V, Gembloux, 1959, p. 175) et H. Silvestre («À propos de la lettre d'Anselme de Laon à Héribrand de Saint-Laurent ", Recherches de théologie ancienne et médiévale, 28, 1961, p. 12-14). Le ms. du Bec, dans lequel se trouvait cette version, a malheureusement disparu, mais, selon Cédric Giraud, que nous remercions pour ce renseignement, on aurait plus affaire à un florilège monastique qu'à une collection de sentences d'origine scolaire. 
topos littéraire, comme le montre déjà la correspondance anonyme d'un moine de l'abbaye du Bec au milieu du XII ${ }^{\mathrm{e}}$ siècle ${ }^{135}$. En réalité, l'autorité de la maxime a été définitivement sapée par la critique scolastique, telle qu'on peut l'observer dans le Décret de Gratien.

Rassemblant l'héritage des collections canoniques, Gratien, qui était luimême un régulier, consacre la première quaestio de sa Causa XVI à réduire les contradictions entre les autorités qui s'étaient exprimées sur le sacerdoce monastique. Après avoir présenté les textes interdisant un tel ministère (c. 1 à 12), il montre que ces mesures visent en fait des cas particuliers (c. 13 à 19) et finit par introduire les distinctions classiques entre moines-prêtres et moines-laïcs, d'une part (c. 21 à 25), et entre pouvoir sacerdotal et juridiction, d'autre part (c. 26 et 27) ${ }^{136}$. Mais, dans le dictum qui suit le canon 39, Gratien tient à revenir sur le précepte hiéronymien ${ }^{137}$. Pour lui, il ne doit pas être considéré comme une proposition universelle, puisqu'il connaît de nombreuses exceptions. Mais, pour ne pas laisser entendre que Jérôme se serait trompé, il suggère que l'intention de l'auteur était seulement de montrer ce qui convenait le mieux à chaque état, et non d'interdire à tous les moines d'assumer des fonctions cléricales. Surtout, il constate qu'à l'époque patristique, il n'y avait pas encore de moine qui fût également clerc, ce qui l'invite à contextualiser la sentence ${ }^{138}$. La plupart des décrétistes qui commentent ce passage reprennent l'argumentation de Gratien, même s'ils ne citent pas toujours l'intégralité du canon ${ }^{139}$. Pour le Bolonais Rufin, qui rédige sa Somme au Décret vers 1160, la maxime de Jérôme visait en fait certains moines, qui quittaient leurs monastères,

${ }^{135}$ Dans la Lettre 16, adressée à un archevêque de Rouen, l'auteur évacue l'objection sans même la discuter, tandis que, dans la Lettre 15, adressée à un reclus nommé Guerric, il n'invoque la maxime que pour mieux l'écarter: Nec praetendatis illud Hieronymi [...] (J. Leclercq, «Les lettres familières d'un moine du Bec», Analecta monastica, $2^{\mathrm{e}}$ sér., Roma [Studia Anselmiana, 31], 1953, p. 170-171). Reprenant le dossier épistolaire édité par Leclercq, Fr. Hudry (Alain de Lille [?], Lettres familières (1167-1170), éd. et trad. Fr. Hudry, Paris, 2003) formule l'hypothèse que ces lettres seraient en fait l'œuvre d'Alain de Lille († 1203), mais Fr. Dolbeau a émis de sérieux doutes sur cette interprétation (compte rendu dans Archivum Latinitatis Medii Aevi, 61, 2003, p. 338-342).

${ }^{136} \mathrm{Ch}$. Dereine, «Le problème de la cura animarum », art. cit., n. 1, p. 308.

${ }^{137}$ Ce dictum figurait déjà dans la première version du Décret, rédigée vers 1139 (A. Winroth, The Making of Gratian's Decretum, op. cit., n. 100, p. 215).

${ }^{138}$ Gratianus, Decretum, C. 16, q. 1, d. p. c. 39: Voluit ergo Jeronimus distinguere inter personam monachi et clerici, ostendens quid cuique ex proprio offitio conveniat [...] Monachos autem usque ad tempus Eusebii, Zosimi et Siricii, monachos simpliciter et non clericos fuisse, ecclesiastica testatur ystoria. Idem etiam Jeronimus refert monachos Scithiae convenisse in unum, ut sacerdotem sibi invenirent, qui eis missarum solempnia celebraret (éd. cit., n. 99, col. 772).

${ }^{139}$ La Somme du Bolonais Paucapalea (vers 1150) reproduit presque mot pour mot le dictum de Gratien (Die Summa des Paucapalea über das Decretum Gratiani, éd. J. Fr. von Schulte, Giessen, 1890, p. 86-87). En revanche, la sentence de Jérôme ne se trouve ni chez Rolandus (éd. Fr. Thaner, Innsbruck, 1874), ni chez Étienne de Tournai (éd. J. Fr. von Schulte, Giessen, 1891), ni dans la Summa Parisiensis (éd. T. P. McLaughlin, Toronto, 1952), ni chez Simon de Bisignano (éd. électronique de P. Aimone : www.unifr.ch/cde/summa_simonis_de.php). 
sans autorisation de l'évêque, pour usurper le ministère paroissial. Le décrétiste précise toutefois que ce n'est plus le cas maintenant et que, la cause d'une telle disposition ayant cessé, l'interdiction elle-même n'a plus lieu d'être ${ }^{140}$. Quant à la Summa Coloniensis, composée à Cologne vers 1169, elle reprend non seulement les arguments de Gratien, mais aussi ceux de Rufin, tout en développant déjà un usage presque proverbial de la sentence ${ }^{141}$. En fait, les canonistes appliquent ici la méthode scolastique typique, qui permet de relativiser une affirmation doctrinale ${ }^{142}$. Comme le rappelle Alain Boureau, les textes patristiques, à l'inverse du canon formulé par le pape ou le concile, n'ont pas d'autorité contraignante : ils préviennent seulement de l'erreur, mais pas de l'inexactitude dans l'élaboration de la vérité ${ }^{143}$. Confrontée aux contradictions des Pères, la critique textuelle devait rechercher l'intention première de l'auteur en replaçant l'affirmation dans son contexte. La portée du discours était ainsi limitée, sans pour autant toucher à la dignité du Père de l’Église. Mais, par le biais de cette distanciation, l'analyse scolastique enlevait toute valeur probatoire directe au texte patristique. Soumise à un tel traitement, la formule de Jérôme ne pouvait donc que perdre sa vertu de sentence.

Le célèbre passage de l'Adversus Vigilantium contestant aux moines la fonction d'enseigner ne fut élevé au rang de sentence qu'à la fin du $\mathrm{XI}^{\mathrm{e}}$ siècle, en Italie centro-septentrionale. Dans un contexte de lutte entre moines et clercs

140 Rufinus, Summa Decretorum, C. 16, q. 1: Illud autem Jeronimi: "Monachus non doctoris " et octo sibi continue subjuncta capitula ex causa intelligenda sunt, videlicet propter temeritatem monachorum quorundam qui, cum nullis parochialibus ecclesiis essent destinati, sine licentia episcopi de monasterio exeuntes seque de sua religiositate jactantes publice predicabant et baptizabant et penitentiam dabant. Talium monachorum improbitas causam dedit edicto, sed causa cessante cessat prohibitio (Rufinus von Bologna, Summa Decretorum, éd. H. Singer, Paderborn, 1902, p. 353-354). On reconnaît là un célèbre adage juridique : Cessante causa, cessat effectus.

${ }^{141}$ La Summa Coloniensis présente la maxime sous la forme du fragment $3(11,3)$, puis résout la contradiction en reproduisant la solution de Rufin $(11,7)$, avant de rappeler les distinctions du dictum de Gratien $(11,12)$ sans toutefois répéter la sentence (Summa «Elegantius in iure diuino » seu Coloniensis, éd. G. Fransen, S. Kuttner, t. III, Città del Vaticano, 1986, p. 126, 128, 130-131). Dans une autre partie de la Somme $(5,105)$, l'auteur réutilise la structure de la maxime pour en faire une sorte d'adage, sans grand rapport avec le sens originel de la sentence : dicimus quod monachus, licet plangentis non litigantis habeat officium [...] (ibid., t. II, p. 101).

${ }^{142}$ Cette démarche correspond exactement à celle que Rupert de Deutz adopte dès 1125, dans son In Regulam sancti Benedicti, 3, 11 (PL 170, col. 519D), à l'égard d'une autre maxime hiéronymienne, extraite de la Lettre 58,5 (éd. cit., n. 60, p. 534, 1. 6-7), où Jérôme rappelle aux moines qu'ils ont pour pères saint Paul, saint Antoine et d'autres moines laïcs de l'Antiquité. À cela Rupert répond simplement que les circonstances ont changé : At nunc alius Ecclesiae status [...] (J. Leclercq, « Nouvelle réponse de l'ancien monachisme aux critiques des Cisterciens ", Revue bénédictine, 67, 1957, p. 94).

${ }^{143}$ A. Boureau, «L'usage des textes patristiques dans les controverses scolastiques », Revue des sciences philosophiques et théologiques, 91, 2007, p. 39-49. 
séculiers, la maxime de Jérôme avait surtout pour but de réserver à ces derniers les attributs du sacerdoce et, au-delà, de leur assurer le contrôle de la réforme de l'Église, qui avait pourtant commencé dans les milieux monastiques. Ce n'était donc pas, à proprement parler, l'enseignement scolaire des moines qui était visé, mais l'usurpation par eux du ministère de la parole. Les canonistes italiens issus des milieux réformateurs ont ainsi transformé le texte hiéronymien en une arme antimonastique, qui avait d'autant plus d'autorité qu'elle provenait de l'un des pères du monachisme. Si la sentence a continué à circuler dans des collections canoniques italiennes jusqu'au Décret de Gratien, c'est néanmoins l'œuvre d'Yves de Chartres qui a contribué à la populariser et à la faire connaître des théologiens. La maxime entre alors pleinement dans les débats ecclésiologiques qui opposent les chanoines réguliers aux tenants de la tradition monastique. Mais c'est toujours le sacerdoce des moines que cherche à légitimer le bénédictin Rupert de Deutz en réfutant systématiquement la sentence. Il reste que, victime de son succès et de l'apaisement de la controverse au milieu du XII ${ }^{\mathrm{e}}$ siècle, la formule de Jérôme finit par perdre toute autorité, sous l'effet de la critique textuelle à laquelle la soumettent les auteurs scolastiques.

La diffusion de ce fragment patristique au tournant des $\mathrm{XI}^{\mathrm{e}}$ et $\mathrm{XII}^{\mathrm{e}}$ siècles montre toute la complexité de la notion d'enseignement. L'enjeu du débat tourne essentiellement autour du sacerdoce et de la prédication des moines. La possibilité pour eux d'entretenir des écoles au sein des monastères ne leur est ainsi jamais contestée. On aurait tort cependant d'exclure cette controverse de l'étude du système d'enseignement médiéval, sous prétexte qu'elle n'intéresserait pas l'éducation scolaire au sens strict. En effet, l'école n'est pas alors distincte de la prédication: interdire aux moines de prêcher, c'est en réalité leur contester le droit d'enseigner publiquement dans l'Église et donc leur refuser l'exercice de ce qu'on considérait, à l'époque, comme l'élément essentiel de l'activité enseignante. Les clercs réformateurs pouvaient parfaitement admettre un enseignement monastique interne, c'est-à-dire privé, tout en refusant aux moines un enseignement public. Il s'agissait pour eux de deux notions distinctes et d'ailleurs dissociées dans les faits, alors que nous associons aujourd'hui communément l'ensemble des activités scolaires au sein d'un même système global. Les $\mathrm{XI}^{\mathrm{e}}$ et $\mathrm{XII}^{\mathrm{e}}$ siècles constituent justement le point de passage vers le modèle actuel, et la réception de la maxime de Jérôme permet de saisir cette évolution des mentalités. Sous la plume de certains théologiens français, elle a pu en effet s'appliquer à l'activité strictement scolaire des moines et même parfois, dans le cas de Roscelin de Compiègne, à leur autorité professorale. On peut donc dire que la mutation que subit cette sentence en passant de l'Italie à la France et du droit canonique à la théologie illustre, à sa manière, l'émergence d'une fonction magistrale propre au sein de la prédication ${ }^{144}$.

Thierry Kouamé Université Paris 1 Panthéon-Sorbonne

LAMOP (UMR 8589)

\footnotetext{
${ }^{144}$ Sur la genèse d'une autorité magistrale au XII ${ }^{\mathrm{e}}$ s., voir, en dernier lieu, C. Giraud, «Per verba magistri », op. cit., n. 103.
} 


\title{
Annexe 1 : Classement des fragments de la sentence de Jérôme
}

\author{
$A V=S$. Hieronymi presbyteri opera, t. III/5, Adversus Vigilantium, éd. J.-L. Feiertag, \\ Turnhout (CCSL, 79C), 2005. \\ Fragment 1 : Monachus non doctoris habet, sed plangentis officium $(A V, 15,1.14-15)$ \\ Fragment 2 : Monachus [...] qui vel se vel mundum lugeat $(A V, 15,1.14-16)$ \\ Fragment 3 : Monachus [...] Domini pavidus praestoletur adventum $(A V, 15,1.14-16)$ \\ Fragment $4:$ Monachus $[\ldots]$ ne impingat et corruat atque frangatur $(A V, 15,1.14-18)$ \\ Fragment 5 : Monachus [...] ut etiam quae tuta sunt pertimescat $(A V, 15,1.14-21)$ \\ Fragment $6:$ Monachus $[\ldots]$ ad illicitos ducat amplexus $(A V, 15,1.14-16,1.4)$ \\ Fragment 7 : Monachus $[\ldots]$ ne perdam aliquando victoriam $(A V, 15,1.14-16,1.8)$
}

\section{Annexe 2 : Occurrences de la sentence aux $\mathrm{XI}^{\mathrm{e}}$ et $\mathrm{XII}^{\mathrm{e}}$ siècles}

v. 1063-85 Collectio CLXXXIII titulorum, 143, $5 \quad$ Fragment 3

1084 Petrus Crassus (?), Defensio Heinrici IV regis, $5 \quad$ Fragment 5

v. 1083-85 Collectio Vlibrorum (Vat. lat. 1348), 3, 28, $3 \quad$ Fragment 3

v. 1086-89 Anselmus Lucensis, Collectio canonum (version A Aucta), 7, 128 Fragment 6

v. 1089 Collectio XIII librorum (Berlin, Savigny 3), 6, $94 \quad$ Fragment 6

$\begin{array}{lll}\text { v. } 1093 & \text { Ivo Carnotensis, Decretum, 7, } 3 & \text { Fragment } 7\end{array}$

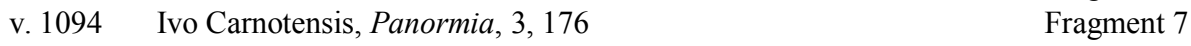

1094 Ivo Carnotensis, Ep. XXXVI Fragment 1

1094 Ivo Carnotensis, Ep. XLI $\quad$ Fragment 2

$\begin{array}{lll}\text { fin } \mathrm{XI}^{\mathrm{e}} \text { s. } & \text { Ivo Carnotensis, Collectio Tripartita, 3, 11,2 } & \text { Fragment } 7\end{array}$

fin $\mathrm{XI}^{\mathrm{e}}$ s. $\quad$ Collectio Sinemuriensis (Orléans, BM 306), App. $680 \quad$ Fragment 3

v. 1101 Ivo Carnotensis, Ep. CVIII Fragment 2

v. 1104-13 Polycarpus, 4, 35, $13 \quad$ Fragment 4

v. 1112-20 Collectio VII librorum (Wien, ÖNB 2186), 5, 52, $1 \quad$ Fragment 3

$\begin{array}{lll}\text { v. } 1120 & \text { Collectio Caesaraugustana, 9, } 7 & \text { Fragment } 4\end{array}$

v. 1120 Roscelinus Compendiensis, Epistola ad Petrum Abaelardum Fragment 3

v. 1119-22 Rupertus Tuitiensis, Altercatio monachi et clerici $\quad$ Fragment 1

$\begin{array}{ll}\text { v. } 1123 \text { Collectio X partium, 3, 52, 3, } 4 & \text { Fragment } 7\end{array}$

v. 1111-24 Collectio III librorum, 2, 29, $12 \quad$ Fragment 3

v. 1123-24 Rupertus Tuitiensis, Epistola ad Everardum abbatem Brunwilrensem Fragment 1

v. 1123-25 Collectio IX librorum (Vat. Arch. S. Pietro C.118), 5, 5, $12 \quad$ Fragment 3

v. 1126-27 Petrus Abaelardus, Sermo XXXIII Fragment 3

v. 1130-32 Honorius Augustodunensis, Questio utrum monachis liceat predicare Fragment 1

v. 1130-32 Honorius Augustodunensis, Quod monachis liceat predicare Fragment 1

v. 1137-38 Petrus Abaelardus, Problemata Heloissae, $14 \quad$ Fragment 3

v. 1139 Gratianus, Decretum, C. 16, q. 1, c. $4 \quad$ Fragment 3

v. 1109-43 Anselmus Lucensis, Collectio canonum (version Bb), App. 33 Fragment 3

v. 1132-45 Idungus Prufeningensis, Argumentum super quatuor questionibus $\quad$ Fragment 2

1146 Bernardus Claraevallensis, Ep. CCCLXV, 1 Fragment 1

$\begin{array}{ll}\text { v. 1100-50 Collectio Ambrosiana II (Ambrosiana I.145 inf.), } 10 & \text { Fragment } 7\end{array}$

v. 1150 Paucapalea, Summa Decreti, C. 16, q. $1 \quad$ Fragment 1

v. 1145-55 Lettres familières d'un moine du Bec (Paris lat. 13575), $15 \quad$ Fragment 1

v. 1155 Idungus Prufeningensis, Dialogus duorum monachorum $\quad$ Fragment 2

v. 1169 Summa Coloniensis, 11,3 Fragment 3 